\title{
In silico profiling of Escherichia coli and Saccharomyces cerevisiae as terpenoid factories
}

\author{
Evamaria Gruchattka ${ }^{1 \dagger}$, Oliver Hädicke ${ }^{2}$, Steffen Klamt ${ }^{2}$, Verena Schütz ${ }^{1,3 \dagger}$ and Oliver Kayser ${ }^{1 * \dagger}$
}

\begin{abstract}
Background: Heterologous microbial production of rare plant terpenoids of medicinal or industrial interest is attracting more and more attention but terpenoid yields are still low. Escherichia coli and Saccharomyces cerevisiae are the most widely used heterologous hosts; a direct comparison of both hosts based on experimental data is difficult though. Hence, the terpenoid pathways of E. coli (via 1-deoxy-D-xylulose 5-phosphate, DXP) and S. cerevisiae (via mevalonate, MVA), the impact of the respective hosts metabolism as well as the impact of different carbon sources were compared in silico by means of elementary mode analysis. The focus was set on the yield of isopentenyl diphosphate (IPP), the general terpenoid precursor, to identify new metabolic engineering strategies for an enhanced terpenoid yield.
\end{abstract}

Results: Starting from the respective precursor metabolites of the terpenoid pathways (pyruvate and glyceraldehyde3-phosphate for the DXP pathway and acetyl-CoA for the MVA pathway) and considering only carbon stoichiometry, the two terpenoid pathways are identical with respect to carbon yield. However, with glucose as substrate, the MVA pathway has a lower potential to supply terpenoids in high yields than the DXP pathway if the formation of the required precursors is taken into account, due to the carbon loss in the formation of acetyl-CoA. This maximum yield is further reduced in both hosts when the required energy and reduction equivalents are considered. Moreover, the choice of carbon source (glucose, xylose, ethanol or glycerol) has an effect on terpenoid yield with non-fermentable carbon sources being more promising. Both hosts have deficiencies in energy and redox equivalents for high yield terpenoid production leading to new overexpression strategies (heterologous enzymes/pathways) for an enhanced terpenoid yield. Finally, several knockout strategies are identified using constrained minimal cut sets enforcing a coupling of growth to a terpenoid yield which is higher than any yield published in scientific literature so far.

Conclusions: This study provides for the first time a comprehensive and detailed in silico comparison of the most prominent heterologous hosts E. coli and S. cerevisiae as terpenoid factories giving an overview on several promising metabolic engineering strategies paving the way for an enhanced terpenoid yield.

Keywords: Terpenoids, Isoprenoids, In silico, Elementary mode analysis, Constrained minimal cut sets, Metabolic engineering, Escherichia coli, Saccharomyces cerevisiae

\section{Background}

Terpenoids are a class of natural products with important medicinal and industrial applications such as artemisinin (antimalarial) or paclitaxel (anticancer) [1,2]. However, several of these bioactive compounds are scarce and produced only in low amounts in plants, which makes the production in their native hosts uneconomical and

\footnotetext{
* Correspondence: Oliver.Kayser@bci.tu-dortmund.de

'Equal contributors

'Technical Biochemistry, Department of Biochemical and Chemical Engineering, TU Dortmund University, Emil-Figge-Str. 66, 44227 Dortmund, Germany

Full list of author information is available at the end of the article
}

environmentally destructive [2]. The total chemical synthesis of many terpenoids is challenging due to their complex structure and neither ecologically nor economically efficient [3-5]. Alternatively, the use of a microbial platform organism for the production of terpenoids may offer the possibility of large-scale, cost-effective and environmentally friendly industrial production independent from climate or cultivation risks. Today, Escherichia coli and Saccharomyces cerevisiae are the most widely used microorganisms for heterologous terpenoid production. They are preferably used due to advanced molecular biology tools, growth speed and their well-established use in

\section{Biomed Central}


industrial biotechnology $[5,6]$. While most studies for the production of carotenoid compounds of lower complexity such as lycopene (an antioxidative carotenoid) have been carried out in E. coli, the trends concerning the production of artemisinin or paclitaxel show a more even distribution between these two hosts with an increasingly more prevalent use of S. cerevisiae [5]. This trend might partly be attributed to the fact that $S$. cerevisiae as an eukaryotic organism has the advantage of being more suitable than E. coli for the expression of membranebound plant cytochrome P450 enzymes along with their respective reductase [2] required for the functionalization of a series of terpenoids [7-9]. A further advantage is the possibility to harness different subcellular compartments [10]. Moreover, yeast can withstand reduced $\mathrm{pH}$ and high osmotic pressure [11] and is not susceptible to phage infections.

The production of terpenoids in both organisms requires a sufficient supply of their common precursor isopentenyl diphosphate (IPP) and its isomer dimethylallyl diphosphate (DMAPP), which are then condensed, cyclized etc. leading to the structural diversity of terpenoids. E. coli and S. cerevisiae differ in their metabolic routes to supply these precursors. The 1-deoxy-D-xylulose 5-phosphate (DXP) pathway of E. coli (often also called 2C-methyl-D-erythriol 4-phosphate pathway, MEP) is fed by pyruvate and glyceraldehyde-3-phosphate whereas in the mevalonate (MVA) pathway of $S$. cerevisiae IPP derives from acetyl-CoA $[12,13]$.

Attempts to engineer the DXP pathway in E. coli mainly focused on the combinatorial overexpression of the rate limiting enzymes encoded by $d x s, i d i, d x r$, ispD and ispF with various ranges of expression levels. Those studies stressed the need to carefully balance the expression of the DXP pathway genes with the heterologous terpenoid forming pathway as well as with the host's overall metabolism [14-21]. Alternatively, various examples show that the introduction of an optimized MVA pathway efficiently increases terpenoid production in E. coli, circumventing the largely unidentified regulations of its native DXP pathway and also decoupling the MVA pathway from its native control in yeast [17,22-25].

Numerous engineering strategies have been applied especially to the MVA pathway in S. cerevisiae [11]. Three particularly successful interventions have been described in literature: i) overexpression of a truncated version of 3-hydroxy-3-methylglutaryl-coenzyme A reductase (HMG1), the key regulatory enzyme of the pathway, devoid of feedback inhibition by farnesyl diphosphate (FPP) [26-28]; ii) downregulation of squalene synthase (ERG9) to reduce the flux draining of FPP to biosynthetically related sterols [29,30]; and iii) expression of a mutant transcription factor (upc2-1) to transcriptionally up-regulate several MVA pathway genes $[7,8,31]$.
A direct in vivo head-to-head comparison of both organisms and terpenoid pathways is difficult to interpret, especially if it is considered that the expression of enzymes of both IPP forming pathways has to be balanced carefully for optimal precursor supply and that E. coli and $S$. cerevisiae differ in their central metabolic networks. However, in silico methods can reveal their theoretical potential and give further insight into the properties of the underlying metabolic networks, thus providing a rational basis for metabolic engineering.

Elementary mode analysis (EMA) is a key methodology to study essential properties of a metabolic network. Elementary modes (EMs) characterize the space of feasible steady-state flux distributions; any such flux distribution can be represented as a non-negative (conic) linear combination of elementary modes. Based on the stoichiometric matrix, the steady-state assumption, thermodynamic constraints (reaction reversibilities) and a non-decomposability constraint, elementary modes can be calculated without the need for kinetic data, $a$ priori measurements, or an objective function [32-36]. EMA has been applied to calculate the overall capacity of a given metabolic network, e.g. the theoretical maximum yield under a given genetic constitution or on different substrates $[32,37]$ in order to estimate the potential efficiency of a biotechnological process. Moreover, it has been successfully used as a basis for the computation of intervention strategies to obtain superior production strains [32,38-41]. Recently, Hädicke et al. [40] introduced the concept of constrained minimal cut sets (cMCSs) to compute knockout strategies for coupled product and biomass synthesis allowing not only the specification of functionalities that are to be disabled in the metabolic network but also of those that have to be preserved. Furthermore, all equivalent knockout strategies to accomplish the same engineering goal are computed, giving the researcher the flexibility to choose the best combination of gene deletions in terms of practical realization. cMCSs comprise and extend e.g. the approach of minimal metabolic functionality [42] that has been successfully applied multiple times in order to identify metabolic engineering strategies [41-44].

In this study, EMA is applied to compare the metabolic networks of E. coli and S. cerevisiae for the production of the common terpenoid precursor IPP by means of carbon stoichiometry, energy and redox equivalent requirements. Further, the potential of the two hosts' central metabolic networks to meet those requirements if the MVA, the DXP or both pathways are operating is analyzed. Moreover, the impact of different industrially relevant carbon sources on IPP production is investigated in silico.

While most studies on terpenoid production mainly focused on engineering the DXP and MVA pathway, only a few studies deal with the identification of engineering 
targets in the two host's central metabolism for an improved precursor supply [31,41,45-50] leaving tremendous room for further improvements. Therefore, the impact of heterologously introduced enzymes is analyzed in silico and knockout targets are computed based on constrained minimal cut sets to give guidelines for metabolic engineering in order to obtain a highly efficient IPP platform organism.

\section{Results and discussion}

This section is conceptually divided into two parts. The first subsection focuses on the analysis of the two terpenoid pathways starting with a comparison of the DXP and MVA pathway independent from their host organism. Thereafter, the stoichiometric potential of $E$. coli and S. cerevisiae to produce the precursor IPP is analyzed. For this purpose, models of the central carbon metabolism were built up and analyzed (65 reactions and 50 metabolites for E. coli and 69 reactions and 60 metabolites for S. cerevisiae, both for growth on glucose, see Methods section and Additional file 1 for details). Subsequently, the optimal flux distributions are characterized and the impact of an interchange of the pathways of E. coli and S. cerevisiae is investigated in silico. The pathway analysis part is completed by analyzing alternative substrates and comparing the identified theoretically optimal yields with available literature data. The results of all these calculations are summarized in Table 1.

The second part of the results section is related to target identification for metabolic engineering. Suitable heterologous overexpression candidates are identified and promising knockout strategies are computed by means of cMCSs.

\section{Terpenoid pathway and metabolic network analysis Stoichiometry of terpenoid pathways}

The stoichiometry of both pathways is initially analyzed independently from the respective host organism. The precursors of the DXP pathway are glyceraldehyde-3phosphate (GAP) and pyruvate (PYR) and the overall stoichiometry of this pathway is given by equation 1 .

$$
\begin{aligned}
& \mathrm{GAP}+\mathrm{PYR}+3 \mathrm{NADPH}+2 \mathrm{ATP} \\
& =\mathrm{IPP}+\mathrm{CO}_{2}+3 \mathrm{NADP}^{+}+2 \mathrm{ADP}
\end{aligned}
$$

The MVA pathway is fed by the carbon precursor acetyl-CoA (AcCoA) and its stoichiometry is given by equation 2 .

$$
\begin{aligned}
& 3 \mathrm{AcCoA}+2 \mathrm{NADPH}+3 \mathrm{ATP} \\
& =\mathrm{IPP}+\mathrm{CO}_{2}+2 \mathrm{NADP}^{+}+3 \mathrm{ADP}
\end{aligned}
$$

Both terpenoid pathways lose one $\mathrm{mol} \mathrm{CO}_{2}$ per mol IPP (C5); therefore they are identical with respect to carbon yield $(5 / 6=0.83)$ when starting from their precursors
GAP (C3) and PYR (C3) or AcCoA (C2). Regarding the demand of ATP and NADPH, they show only minor differences as the DXP pathway needs one mol more NADPH but one mol less ATP than the MVA pathway. Hence, a priori, one could assume that no significant differences in achievable IPP yields occur between E. coli and S. cerevisiae.

\section{Metabolic potential of wild type E. coli and S. cerevisiae to supply IPP}

Both terpenoid pathways are now analyzed with respect to the metabolic background of their respective host. A first comparison of the maximal IPP yields purely based on carbon stoichiometry, ignoring energy and redox equivalent requirements (i.e. when ATP and $\mathrm{NAD}(\mathrm{P}) \mathrm{H}$ are initially considered to be external metabolites in the model), shows a significant difference. With energy and redox equivalents in excess, the maximum IPP yield on glucose via the DXP pathway in E. coli is $5 / 6=0.83 \mathrm{C}$ $\mathrm{mol} / \mathrm{C}$-mol compared to $5 / 9=0.56 \mathrm{C}$ - $\mathrm{mol} / \mathrm{C}$-mol in S. cerevisiae if the MVA pathway is used (Figure 1). This is due to different amounts of carbon loss via $\mathrm{CO}_{2}$ in the formation of the terpenoid pathway precursors. The formation of one mol acetyl-CoA, the precursor of the MVA pathway, includes the generation (and thus carbon loss) of one mol $\mathrm{CO}_{2}$. In contrast, the formation of one mol pyruvate and one mol glyceraldehyde-3-phosphate, the precursors of the DXP pathway, does not involve a carbon loss via $\mathrm{CO}_{2}$.

Besides the different carbon use efficiencies, the energy and redox equivalent requirements for IPP synthesis differ in E. coli and S. cerevisiae as well (Figure 1). For the formation of one mol IPP from glucose (GLC) in E. coli, the overall stoichiometry is given by equation 3.

$$
\begin{aligned}
& \mathrm{GLC}+2 \mathrm{ATP}+3 \mathrm{NADPH}+\mathrm{NAD}^{+} \\
& =\mathrm{IPP}+\mathrm{CO}_{2}+2 \mathrm{ADP}+3 \mathrm{NADP}^{+}+\mathrm{NADH}
\end{aligned}
$$

In S. cerevisiae, the overall stoichiometry for synthesizing one mol IPP with glucose as substrate via the MVA pathway (considering only the constitutive $\mathrm{NADP}^{+}$dependent aldehyde dehydrogenase, $A L D 6$ ) is given by equation 4.

$$
\begin{aligned}
& 1.5 \mathrm{GLC}+3 \mathrm{ATP}+\mathrm{NADP}^{+}+3 \mathrm{NAD}^{+} \\
& =\mathrm{IPP}+4 \mathrm{CO}_{2}+3 \mathrm{AMP}+\mathrm{NADPH}+3 \mathrm{NADH}
\end{aligned}
$$

Now, in the context of the metabolic background, significant differences are observed. When the pathways are considered isolated from the network, they can both serve as redox sinks as they require reduced redox equivalents (see equations 1 and 2). However, due to the synthesis of the specific pathway precursors, different energy and redox equivalent requirements 
Table 1 Overview of in silico computations: number of elementary modes (EMs) and maximal IPP and biomass carbon yields on different carbon sources [C-mol/C-mol] for computations for E. coli and S. cerevisiae

\begin{tabular}{|c|c|c|c|c|c|c|}
\hline Organism & $\begin{array}{l}\text { Carbon } \\
\text { source }\end{array}$ & Characteristics & EMs & $\begin{array}{l}\text { Max IPP yield at } \\
\text { zero growth }\end{array}$ & $\begin{array}{l}\text { Max IPP yield } \\
\text { with growth }\end{array}$ & $\begin{array}{l}\text { Max biomass } \\
\text { carbon yield }\end{array}$ \\
\hline E. coli & Glucose & Wild type & 36,590 & 0.67 & 0.57 & 0.85 \\
\hline E. coli & Glucose & Wild type, anaerobic & 8,343 & 0.48 & 0.41 & 0.35 \\
\hline E. coli & Glucose & ATP source & 48,603 & 0.71 & 0.57 & 0.99 \\
\hline E. coli & Glucose & NADH source & 45,570 & 0.83 & 0.57 & 0.99 \\
\hline E. coli & Glucose & NADPH source & 44,842 & 0.83 & 0.57 & 0.99 \\
\hline E. coli & Glucose & GAP-DH $\left(\mathrm{NADP}^{+}\right)$ & 74,651 & 0.71 & 0.62 & 0.99 \\
\hline E. coli & Glucose & MVA only & 35,968 & 0.56 & 0.54 & 0.85 \\
\hline E. coli & Glucose & $\mathrm{MVA}+\mathrm{DXP}$ & 44,850 & 0.67 & 0.59 & 0.85 \\
\hline E. coli & Glycerol & Wild type & 26,160 & 0.78 & 0.77 & 0.99 \\
\hline E. coli & Glycerol & ATP source & 29,088 & 0.83 & 0.77 & 0.99 \\
\hline E. coli & Glycerol & NADH source & 29,126 & 0.83 & 0.77 & 0.99 \\
\hline E. coli & Glycerol & NADPH source & 28,252 & 0.83 & 0.77 & 0.99 \\
\hline E. coli & Glycerol & GAP-DH $\left(\mathrm{NADP}^{+}\right)$ & 62,665 & 0.83 & 0.77 & 0.99 \\
\hline E. coli & Glycerol & MVA only & 22,225 & 0.56 & 0.45 & 0.99 \\
\hline E. coli & Glycerol & $M V A+D X P$ & 28,326 & 0.78 & 0.77 & 0.99 \\
\hline S. cerevisiae & Glucose & Wild type & 9,844 & 0.53 & 0.39 & 0.80 \\
\hline S. cerevisiae & Glucose & Wild type, anaerobic & 495 & 0.10 & 0.09 & 0.21 \\
\hline S. cerevisiae & Glucose & ATP source & 15,326 & 0.56 & 0.39 & 0.89 \\
\hline S. cerevisiae & Glucose & $\mathrm{NADH}$ source & 12,080 & 0.56 & 0.39 & 0.89 \\
\hline S. cerevisiae & Glucose & $\mathrm{PDH}$ & 17,943 & 0.56 & 0.51 & 0.81 \\
\hline S. cerevisiae & Glucose & Mitochondrial MVA only & 10,232 & 0.56 & 0.51 & 0.80 \\
\hline S. cerevisiae & Glucose & Mitochondrial + cytosolic MVA & 128,427 & 0.56 & 0.53 & 0.82 \\
\hline S. cerevisiae & Glucose & $\mathrm{ACL}$ & 17,155 & 0.55 & 0.39 & 0.80 \\
\hline S. cerevisiae & Glucose & DXP only & 8,865 & 0.64 & 0.57 & 0.80 \\
\hline S. cerevisiae & Glucose & $\mathrm{DXP}+\mathrm{MVA}$ & 11,738 & 0.64 & 0.57 & 0.80 \\
\hline S. cerevisiae & Glucose & DXP + ATP source & 13,731 & 0.66 & 0.57 & 0.89 \\
\hline S. cerevisiae & Glucose & $\mathrm{DXP}+\mathrm{NADH}$ source & 10,714 & 0.66 & 0.57 & 0.89 \\
\hline S. cerevisiae & Glucose & DXP + NADPH source & 16,754 & 0.80 & 0.57 & 0.89 \\
\hline S. cerevisiae & Glucose & $\mathrm{DXP}+\mathrm{TH}$ & 25,254 & 0.67 & 0.57 & 0.81 \\
\hline S. cerevisiae & Glucose & $\mathrm{DXP}+\mathrm{GAP}-\mathrm{DH}\left(\mathrm{NADP}^{+}\right)$ & 28,383 & 0.67 & 0.57 & 0.81 \\
\hline S. cerevisiae & Galactose & Wild type & 9,844 & 0.53 & 0.39 & 0.80 \\
\hline S. cerevisiae & Fructose & Wild type & 9,844 & 0.53 & 0.39 & 0.80 \\
\hline S. cerevisiae & Xylose & $X I$ & 6,330 & 0.53 & 0.39 & 0.80 \\
\hline S. cerevisiae & Xylose & $\mathrm{XR}-\mathrm{XDH}$ & 16,911 & 0.53 & 0.51 & 0.80 \\
\hline S. cerevisiae & Xylose & $X I+$ DXP only & 7,364 & 0.64 & 0.42 & 0.80 \\
\hline S. cerevisiae & Xylose & $X I+D X P+M V A$ & 9,088 & 0.64 & 0.44 & 0.80 \\
\hline S. cerevisiae & Xylose & $X R-X D H+D X P$ only & 16,513 & 0.64 & 0.42 & 0.80 \\
\hline S. cerevisiae & Xylose & $X R-X D H+D X P+M V A$ & 20,337 & 0.64 & 0.51 & 0.80 \\
\hline S. cerevisiae & Glycerol & Wild type & $2,648,133$ & 0.56 & 0.47 & 0.87 \\
\hline S. cerevisiae & Glycerol & Wild type w/o glyoxylate cycle & 5,377 & 0.56 & 0.35 & 0.87 \\
\hline S. cerevisiae & Glycerol & DXP only & $2,401,411$ & 0.67 & 0.60 & 0.87 \\
\hline S. cerevisiae & Glycerol & $\mathrm{DXP}+\mathrm{MVA}$ & $4,038,007$ & 0.67 & 0.60 & 0.87 \\
\hline S. cerevisiae & Glycerol & DXP + NADPH source & $3,735,984$ & 0.83 & 0.80 & 0.94 \\
\hline
\end{tabular}


Table 1 Overview of in silico computations: number of elementary modes (EMs) and maximal IPP and biomass carbon yields on different carbon sources [C-mol/C-mol] for computations for E. coli and S. cerevisiae (Continued)

\begin{tabular}{|c|c|c|c|c|c|c|}
\hline S. cerevisiae & Glycerol & $\mathrm{DXP}+\mathrm{GAP}-\mathrm{DH}\left(\mathrm{NADP}^{+}\right)$ & $6,153,971$ & 0.76 & 0.63 & 0.90 \\
\hline S. cerevisiae & Ethanol & Wild type & $2,874,235$ & 0.68 & 0.59 & 0.78 \\
\hline S. cerevisiae & Ethanol & ATP source & $3,559,725$ & 0.83 & 0.59 & 0.81 \\
\hline S. cerevisiae & Ethanol & NADH source & $3,635,994$ & 0.83 & 0.62 & 0.81 \\
\hline S. cerevisiae & Ethanol & DXP only & $2,846,217$ & 0.63 & 0.57 & 0.78 \\
\hline S. cerevisiae & Ethanol & $\mathrm{DXP}+\mathrm{MVA}$ & $3,133,493$ & 0.68 & 0.59 & 0.78 \\
\hline
\end{tabular}

GAP-DH $\left(\mathrm{NADP}^{+}\right)=\mathrm{NADP}^{+}$-dependent glyceraldehyde-3-phosphate dehydrogenase; $\mathrm{PDH}=$ cytosolic pyruvate dehydrogenase complex; $\mathrm{ACL}=\mathrm{ATP}$-citrate-lyase; $\mathrm{TH}=$ soluble and energy-independent transhydrogenase; $\mathrm{XI}=$ xylose isomerase pathway; XR-XDH =xylose reductase and xylitol dehydrogenase pathway

have to be balanced by the organism's metabolism. In E. coli, the synthesis of IPP is still accompanied by oxidation of NADPH (equation 3) whereby in S. cerevisiae reduced redox equivalents are generated (equation 4). In both organisms, additional ATP has to be generated from the substrate for IPP synthesis, which leads to a further reduction of the maximum IPP yield.

Taking the cofactors explicitly into account requires a network-wide view of all pathways. Therefore, elementary mode analysis was performed on the metabolic networks of E. coli (DXP pathway) and S. cerevisiae (MVA pathway) wild types with glucose as the single carbon source. In total, 36,590 elementary modes were obtained for the E. coli network and 9,844 for the S. cerevisiae network (Figure 2). Of these, 2,597 modes (26.4\%) of S. cerevisiae and even 7,675 modes (21.0\%) of E. coli include IPP formation. Computed elementary modes exhibit different yields for IPP and biomass in both organisms (Figure 2). The maximum IPP yield for $E$. coli is $0.67 \mathrm{C}$-mol/C-mol and $0.53 \mathrm{C}$-mol/C-mol for $S$. cerevisiae. If only elementary modes that couple IPP synthesis with biomass formation are considered, the maximum IPP yield drops to 0.57 C-mol/C-mol in E. coli and $0.39 \mathrm{C}$-mol/C-mol in S. cerevisiae, respectively.

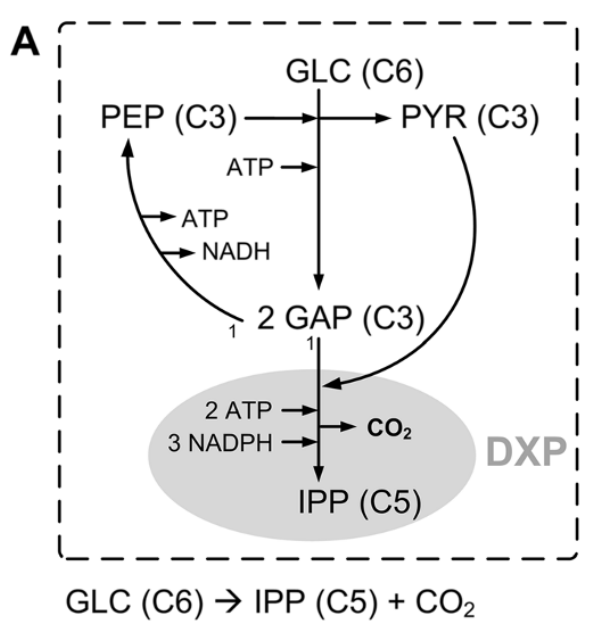

IPP/GLC = 0.83 C-mol/C-mol

\section{$\sum 1 \mathrm{NADH}$ produced 3 NADPH needed \\ 2 ATP needed}

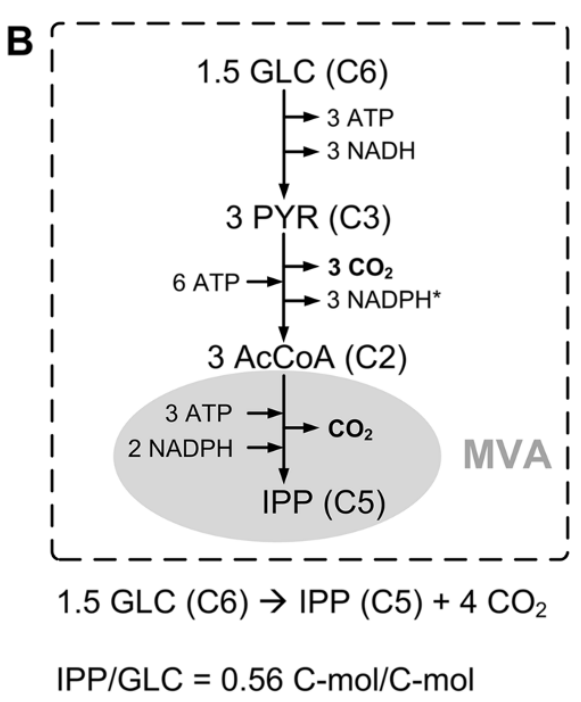

\section{$\Sigma 3 \mathrm{NADH}$ produced 1 NADPH produced 6 ATP needed}

Figure 1 Comparison of the DXP and MVA pathway. A section of the central carbon metabolism from glucose (GLC) to IPP: A: of E. coli including glycolysis and DXP pathway, and $\mathbf{B}$ : of S. cerevisiae including glycolysis and the MVA pathway. The maximal IPP yields based on carbon stoichiometry and ignoring energy and redox equivalent requirements $(5 / 6=0.83$ and $5 / 9=0.56 \mathrm{C}-\mathrm{mol} / \mathrm{C}-\mathrm{mol})$ differ due to different carbon loss via $\mathrm{CO}_{2}$ in the formation of the precursors pyruvate (PYR) and glyceraldehyde-3-phosphate (GAP) or acetyl-CoA (AcCoA). The sum shows energy requirements and redox equivalents that have to be balanced by the organism's metabolism. *: Note that cytosolic NADP ${ }^{+}$-dependent aldehyde dehydrogenase (ALD6) is constitutive while cytosolic NAD+-dependent aldehyde dehydrogenases (ALD2, ALD3) are stress-induced, glucoserepressed [51] and were not considered. 

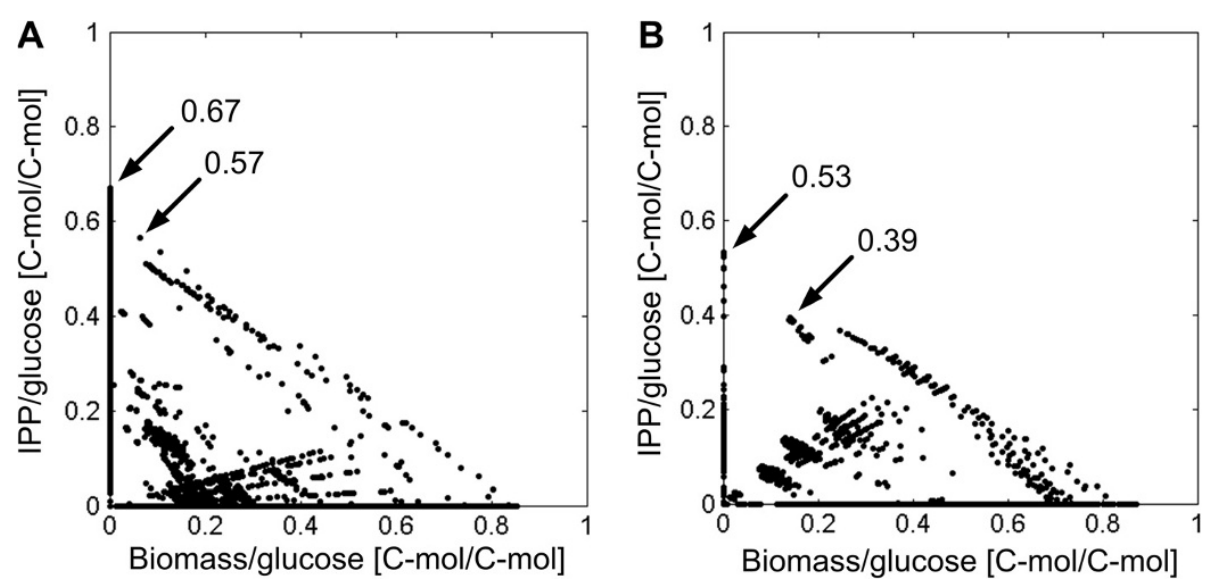

Figure 2 Comparison of the solution space spanned by elementary modes. A: Wild type network of E. coli (36,590 EMs). B: Wild type network of $S$. cerevisiae (9,844 EMs). The biomass yield on glucose is plotted against the IPP yield on glucose [C-mol/C-mol] for all computed elementary modes. Elementary modes on the axes represent modes producing either only biomass ( $x$-axis) or only IPP (y-axis). Theoretical maximal IPP yields at zero growth as well as with biomass formation are highlighted.

Hence, E. coli has a higher theoretical potential to provide IPP, the common terpenoid precursor.

\section{Comparison of flux distributions with maximum IPP production}

The differences in carbon stoichiometry of both IPP forming pathways, in energy and redox equivalent requirements as well as in the two hosts' central carbon metabolisms lead to different theoretical maximal IPP yields. A comparison of the theoretical flux distributions in both organisms provides a detailed picture of the reactions involved and reveals key pathways contributing to maximum IPP production as well as pathways that could be dispensable. Furthermore, they show limitations and provide valuable starting points for metabolic engineering. For E. coli and S. cerevisiae, two and five elementary modes enable the optimum yield, respectively. In this subsection, main characteristics of these optimal flux distributions are discussed. The flux maps of one particular optimal mode for each case are shown in Figures 3 and 4, respectively.

Yield optimal flux distributions in E. coli In E. coli, 3 NADPH as well as 2 ATP are needed for the production of one mol IPP from one mol glucose (see above). In the optimal case, NADPH is provided by a transhydrogenase, an enzyme which transfers reducing equivalents between two nucleotide acceptors to balance the intracellular redox potential $\left(6 \mathrm{NADH}+6 \mathrm{NADP}^{+}+2 \mathrm{ATP}=6 \mathrm{NADPH}+6\right.$ $\left.\mathrm{NAD}^{+}+2 \mathrm{ADP}\right)$. However, this leads to an increased requirement for ATP and NADH. Thus, the cell is forced to branch off some carbon flux to the citric acid cycle to generate NADH and ATP as shown in the optimal flux distributions (Figure 3). NADPH is not provided by the oxidative part of the pentose phosphate pathway in this case due to increased carbon loss via $\mathrm{CO}_{2}$ which leads to decreased IPP yields. The main flux passes through glycolysis and DXP pathway, while a portion passes pyruvate dehydrogenase complex and citric acid cycle to generate ATP, NADH and NADPH along with respiratory chain and transhydrogenase. The high branchoff leads to a carbon loss and thus to a reduction in the maximum IPP yield from 0.83 (if carbon stoichiometry is considered only) to about $0.67 \mathrm{C}$ - $\mathrm{mol} / \mathrm{C}$ - $\mathrm{mol}$ in the optimal flux distributions (Figure 3, see also Table 1). Some fluxes are flexible within optimal elementary modes namely those of malate dehydrogenase, malate quinone oxidoreductase as well as NADH dehydrogenase II indicating a certain flexibility in redox balancing which does not influence the IPP yield. Optimal IPP production does not involve any by-product formation except $\mathrm{CO}_{2}$ and is purely respirative. However, fermentative modes show high product yields as well, up to $0.48 \mathrm{C}$-mol/C-mol.

The limitation in redox equivalents and energy for high yield IPP production can be validated in silico via the introduction of additional artificial ATP, NADH or NADPH sources in the metabolic network model. The introduction of an artificial ATP source increases the maximum yield slightly to 0.71 , while artificial NADH or NADPH sources lead to the theoretical maximum value of about $0.83 \mathrm{C}-\mathrm{mol} / \mathrm{C}-\mathrm{mol}$ (as discussed in the previous section).

Yield optimal flux distributions in S. cerevisiae In S. cerevisiae, $3 \mathrm{NADH}$ and one NADPH are produced via glycolysis while 6 ATP are needed for the production of one mol IPP from 1.5 moles glucose as described above. Generated NADH can be reoxidized via the respiratory chain to produce ATP. However, not enough ATP can be produced due to the stoichiometry of the respiratory 


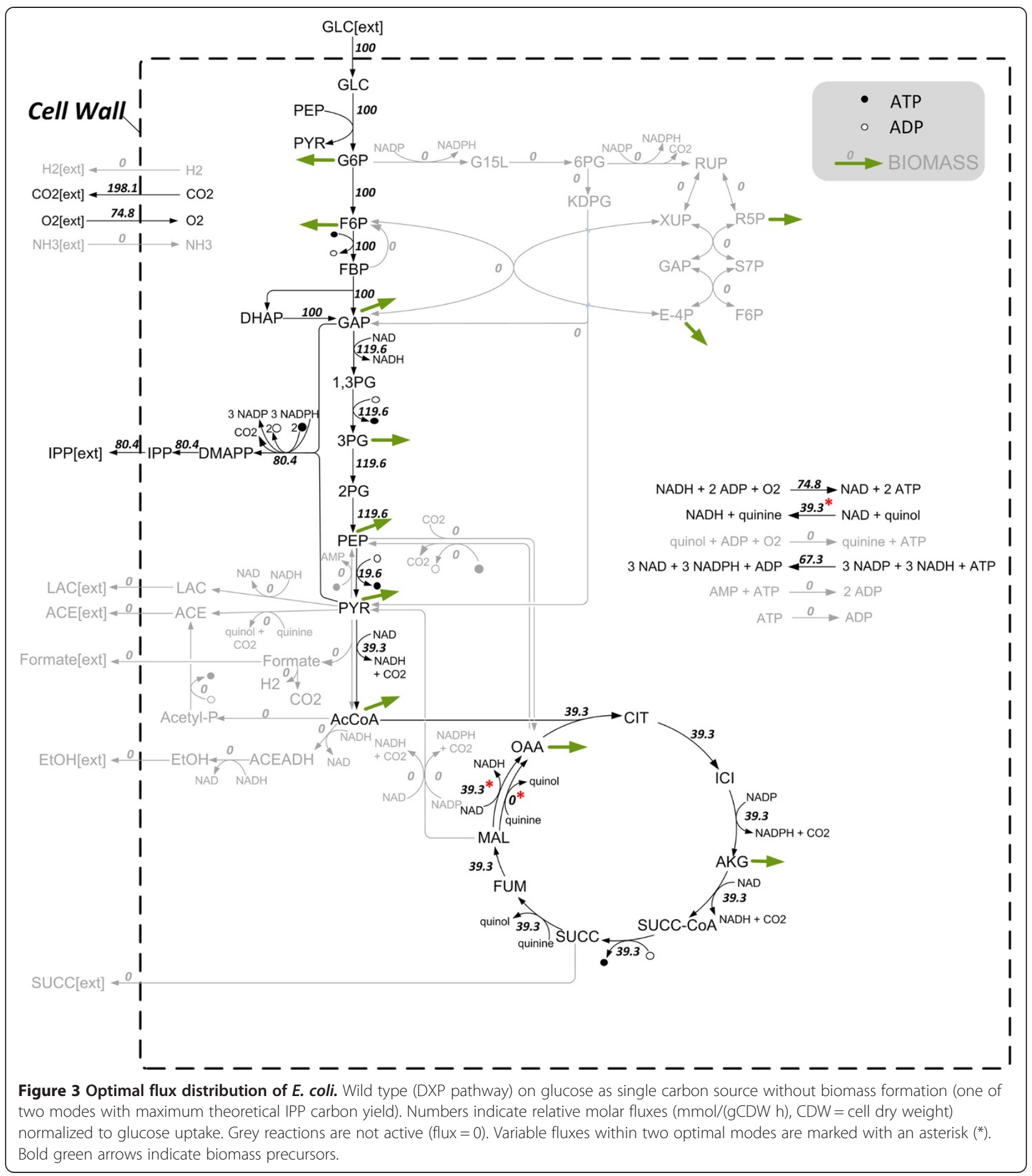

chain. Thus, the cell would have a deficiency in energy for high yield IPP production and would be forced to branch off some carbon flux to the citric acid cycle to generate ATP via NADH. This branch-off is shown in the computed optimal flux distributions (Figure 4). The main flux passes glycolysis, pyruvate dehydrogenase bypass and MVA pathway, while a small part is branched off to pyruvate dehydrogenase complex and citric acid cycle in the mitochondria to generate $\mathrm{NADH}$ which is then reoxidized via the respiratory chain in order to produce ATP. This branch-off leads to a further carbon loss and thus to a reduction of the IPP yield to about $0.53 \mathrm{C}-\mathrm{mol} /$ $\mathrm{C}$-mol in the optimal flux distributions. As shown above, the energy deficiency preventing high yield IPP production 


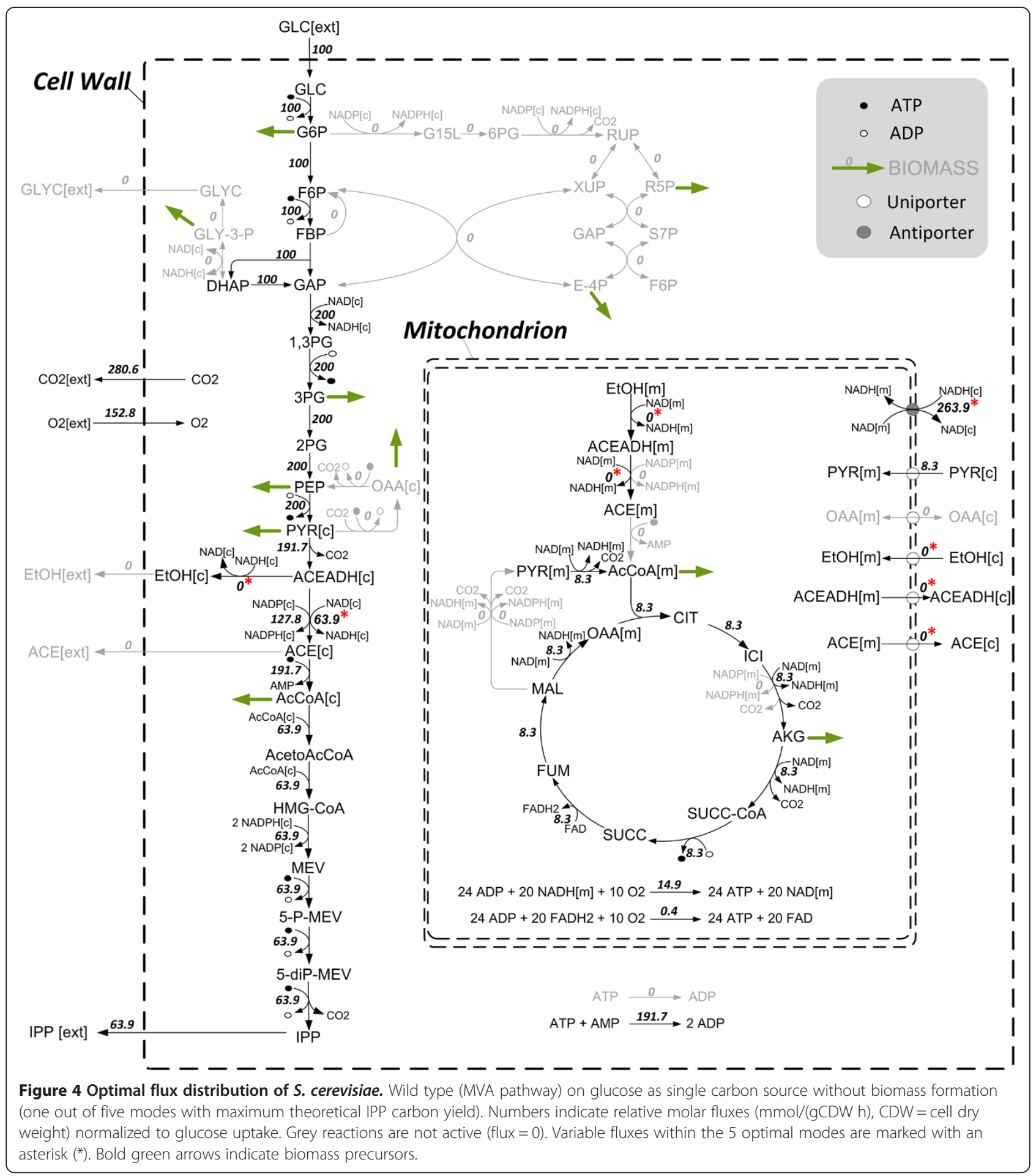

of the MVA pathway can be validated via the introduction of an additional artificial ATP or NADH source in silico which leads to the theoretical maximum value of about $0.56 \mathrm{C}-\mathrm{mol} / \mathrm{C}$-mol. Some fluxes are flexible within optimal elementary modes namely those of mitochondrial and cytosolic alcohol dehydrogenases and $\mathrm{NAD}^{+}$-dependent aldehyde dehydrogenases as well as mitochondrial ethanol, acetaldehyde and acetate transport and a redox shuttle indicating a certain flexibility in NADH redox balancing which does not influence the IPP yield. The optimal flux distributions are purely respirative modes. Respiro-fermentative modes (modes that include respiration as well as the formation of fermentation products) show high product yields as well. However, pure 
fermentative modes are characterized by a very low theoretical maximum product yield of about $0.1 \mathrm{C}$-mol/C-mol. High ATP requirements of the MVA pathway cannot be delivered by fermentative metabolism. Additionally, the formation of $\mathrm{CO}_{2}$, the only side-product, is much higher than in optimal flux distributions in E. coli reflecting the high carbon loss in the formation of acetyl-CoA.

Even though theoretical maximal yields differ in $E$. coli and $S$. cerevisiae, the main flux in computed optimal flux distributions at maximum IPP yield is similar. Key pathways are glycolysis and terpenoid pathway and a portion of the flux is directed to citric acid cycle and respiratory chain. Transhydrogenase is active in E. coli while pyruvate dehydrogenase bypass is active in yeast to supply NADPH. Dispensable reactions include by-product formation except $\mathrm{CO}_{2}$, gluconeogenetic and anaplerotic reactions as well as pentose phosphate pathway if no biomass is produced. Limitations in redox equivalents and energy for high yield IPP production could be validated in silico.

\section{Exchange of terpenoid pathways}

The MVA pathway from $S$. cerevisiae has been functionally introduced in E. coli and this strain produced substantially more terpenoid than strains with the native or even engineered DXP pathway [17]. Due to this observation the MVA pathway was introduced in silico into $E$. coli to analyze its effects on IPP production. The introduction of the MVA and the removal of the DXP pathway $(35,968 \mathrm{EMs})$ lead to a theoretical maximum value of about $0.56 \mathrm{C}$-mol/C-mol (which is slightly higher than the value of 0.53 obtained for $S$. cerevisiae). No energy or redox equivalent limitations exist for high yield IPP production. E. coli possesses a cytosolic pyruvate dehydrogenase complex instead of a pyruvate dehydrogenase bypass (NADPH source). However, transhydrogenase, Entner-Doudoroff pathway as well as malic enzyme can act as NADPH source. Nevertheless, the sole use of the MVA pathway in $E$. coli is stoichiometrically not efficient as the maximum yield is lower than with the native DXP pathway. The coexistence of both pathways in $E$. coli (44,850 EMs, 1,207 of which that use both pathways simultaneously) leads to a theoretical maximum IPP yield of $0.67 \mathrm{C}-\mathrm{mol} / \mathrm{C}$-mol, which is identical to the value if only the DXP pathway is active. However, the theoretical maximum value in an elementary mode, which includes biomass formation, is slightly enhanced to $0.59 \mathrm{C}-\mathrm{mol} /$ C-mol. Thus, the coexistence of both pathways is not only beneficial due to the decoupling of the MVA pathway from its native control in yeast but also in terms of stoichiometry.

In silico, the introduction of the DXP and the removal of the MVA pathway in S. cerevisiae (8,865 EMs) lead to an increase in the theoretical maximum IPP yield to about 0.64 C-mol/C-mol, and 0.57 C-mol/C-mol including biomass formation. The coexistence of both pathways in yeast (11,738 EMs, 276 of which that use both pathways simultaneously) does not lead to a further enhanced IPP yield. Solely the number of elementary modes and thus the flexibility is enhanced. The IPP carbon yield is slightly lower than that one of $E$. coli as yeast does not possess a transhydrogenase to supply NADPH. Hence, pentose phosphate pathway has to deliver NADPH which involves a carbon loss. Pyruvate dehydrogenase bypass can deliver NADPH in the formation of acetyl-CoA; however, acetyl-CoA is not needed for the DXP pathway. The limitation in energy and redox equivalents can be validated in silico by the introduction of artificial ATP, NADH and especially NADPH sources in the model, which lead to an enhanced theoretical maximum IPP yield of 0.66-0.8 C-mol/C-mol (see Table 1).

In conclusion, the heterologous introduction of the MVA pathway, in addition to the DXP pathway, into E. coli as well as the introduction of the DXP pathway into $S$. cerevisiae are theoretically beneficial for high yield IPP production. Although, the DXP pathway has not been functionally expressed in $S$. cerevisiae yet $[13,52]$, it is a promising target for further research.

\section{Comparison of carbon sources}

Studies showed that different carbon sources might have a different potential to supply high product yields $[8,17,25]$. At present, glucose is the most widely used feedstock in bioprocesses [53]. However, other carbon sources are of industrial interest and could serve as substrate for terpenoid production. Molasses consists mainly of sucrose, a disaccharide composed of glucose and fructose, and is a commonly used carbon source [54]. Hemicellulosic hydrolysates of agricultural byproducts like sugarcane bagasse or wood consist mainly of xylose and glucose as well as other sugars like galactose [55] and could be a cheap alternative carbon source for bioprocesses [56]. Pure glycerol is used in different industries too and crude glycerol could be a cheap alternative as it is a by-product from biodiesel production with decreasing price $[53,57]$. Thus, EMA was further performed for glycerol for E. coli. Moreover, the carbon sources galactose, fructose and xylose were analyzed for $S$. cerevisiae as well as glycerol and ethanol because high terpenoid yields have been described with this substrate [8]. The metabolic networks of the non-fermentable carbon sources ethanol and glycerol involve the glyoxylate cycle and an increased number of mitochondrial shuttles in yeast.

Alternative substrate for $\mathbf{E}$. coli The maximum IPP yield in $E$. coli on the non-fermentable carbon source 
glycerol purely based on carbon stoichiometry (ATP and $\mathrm{NAD}(\mathrm{P}) \mathrm{H}$ considered as external metabolites) is identical to that on glucose $(0.83 \mathrm{C}$-mol/C-mol). The overall stoichiometry for the formation of one mol IPP from glycerol is given by equation 5 .

$$
\begin{aligned}
& 2 \mathrm{GLYC}+2 \mathrm{ATP}+\mathrm{NADPH}+\mathrm{NAD}^{+} \\
& =\mathrm{IPP}+\mathrm{CO}_{2}+2 \mathrm{ADP}+\mathrm{NADP}^{+}+\mathrm{NADH}
\end{aligned}
$$

The carbon loss for the formation of IPP from glycerol is identical to glucose. However, less NADPH is required as it is supplied by glycerol-3-phosphate dehydrogenase. If the balances of ATP, NADH and NADPH are taken into account, the yields for E. coli and the native DXP pathway on glycerol (26,160 EMs) were enhanced in comparison to glucose in silico $(0.78 \mathrm{C}$-mol/C-mol at zero growth and $0.77 \mathrm{C}$-mol/C-mol in an elementary mode that includes biomass formation). Yields were slightly lower for the heterologous MVA pathway $(0.56 \mathrm{C}$-mol/C-mol at zero growth and $0.45 \mathrm{C}$-mol/C-mol including biomass formation). Thus, glycerol and the native DXP pathway have the highest potential for E. coli to support high IPP production. The main flux in optimal flux distributions passes lower part of glycolysis and DXP pathway, while only a small portion is diverted to citric acid cycle, respiratory chain and transhydrogenase to generate NADPH, NADH and ATP. The limitation in energy and redox equivalents for high yield IPP production can be validated in silico by the introduction of artificial ATP, NADH or NADPH sources in the model, which lead to the maximum IPP yield of $0.83 \mathrm{C}$-mol/C-mol (see Table 1 ).

Alternative substrates for S. cerevisiae The carbon as well as the overall stoichiometry for the formation of one mol IPP from galactose or fructose is identical to glucose (see equation 4). Accordingly, the numbers of elementary modes as well as the IPP and biomass yields with galactose and fructose are identical to those with glucose. A heterologous pathway has to be introduced into $S$. cerevisiae for xylose as wild type strains are not able to grow on the pentose xylose. Two different pathways have been used for ethanol production based on xylose with yeast: the xylose isomerase (XI) pathway as well as the xylose reductase and xylitol dehydrogenase (XR-XDH) pathway [58]. The carbon stoichiometry for the formation of one mol IPP from xylose is identical to glucose (maximum $0.56 \mathrm{C}-\mathrm{mol} / \mathrm{C}-\mathrm{mol}$ ). Likewise, the overall stoichiometry is identical to the one on glucose for the XI pathway except that $1.8 \mathrm{~mol}$ xylose (XYL) is needed per mol IPP (equation 6).

$$
\begin{aligned}
& 1.8 \mathrm{XYL}+6 \mathrm{ATP}+\mathrm{NADP}^{+}+3 \mathrm{NAD}^{+} \\
& =\mathrm{IPP}+4 \mathrm{CO}_{2}+6 \mathrm{ADP}+\mathrm{NADPH}+3 \mathrm{NADH}
\end{aligned}
$$

The overall stoichiometry is identical for the $\mathrm{XR}-\mathrm{XDH}$ pathway if xylose reductase uses NADH (as xylitol dehydrogenase delivers NADH) and slightly changes if the enzyme uses NADPH. The theoretical maximal IPP and biomass yields are identical to the values on glucose for both the XI (6,330 EMs) and the XR-XDH pathway $(16,911 \mathrm{EMs})$. However, the IPP yield including biomass formation is enhanced to $0.51 \mathrm{C}-\mathrm{mol} / \mathrm{C}$-mol with the XR-XDH pathway. The number of elementary modes and thus the flexibility of the metabolic network is greatly enhanced if the XR-XDH pathway is chosen. The optimal flux distributions were basically identical to those on glucose except that xylose enters glycolysis via the non-oxidative part of pentose phosphate pathway.

The maximum IPP yield on the non-fermentable carbon source glycerol (GLYC) purely based on carbon stoichiometry (ignoring energy and redox equivalents) is with $0.56 \mathrm{C}$-mol/C-mol identical to glucose. However, the overall stoichiometry is slightly modified (equation 7).

$$
\begin{aligned}
& 3 \mathrm{GLYC}+3 \mathrm{ATP}+\mathrm{NADP}^{+}+6 \mathrm{NAD}^{+} \\
& =\mathrm{IPP}+4 \mathrm{CO}_{2}+3 \mathrm{ADP}+\mathrm{NADPH}+6 \mathrm{NADH}
\end{aligned}
$$

Less energy is required and more NADH is delivered for the production of one mol IPP from glycerol. Glycerol is channeled into glycolysis via glyceraldehyde-3phosphate and the main flux passes lower part of glycolysis, pyruvate dehydrogenase bypass and the MVA pathway. The carbon flux is not diverted to citric acid cycle as enough energy is produced via NADH and respiratory chain. Thus, the maximum IPP yield of 0.56 $\mathrm{C}-\mathrm{mol} / \mathrm{C}$-mol is achieved, which is identical to the maximum IPP yield based on carbon stoichiometry only. No limitations in energy or redox equivalent requirements thus exist for high yield IPP production. The optimal flux distributions further reveal that pentose phosphate pathway, side product formation, the first part of citric acid cycle as well as glyoxylate cycle are not active. Some fluxes are flexible namely those of alcohol dehydrogenases, $\mathrm{NAD}^{+}$-dependent aldehyde dehydrogenases, second part of citric acid cycle as well as mitochondrial transport systems indicating a certain flexibility in NADH redox balancing which does not influence the IPP yield. The very high number of elementary modes (2,648,133 EMs) implies an increased redundancy and thus network flexibility and is due to the activity of the glyoxylate cycle. The glyoxylate cycle showed only a weak activity in yeast cells grown on glycerol in our experiments (data not shown). If the cycle is excluded from the computations, the number of elementary modes decreases (5,377 EMs) but the theoretical maximum IPP yield remains 0.56 $\mathrm{C}-\mathrm{mol} / \mathrm{C}$-mol. Nevertheless, the IPP yield including biomass formation is much higher if the cycle is included 
(0.47 C-mol/C-mol versus $0.35 \mathrm{C}$-mol/C-mol) indicating that an active glyoxylate cycle could be of benefit.

The maximum IPP yield on the non-fermentable carbon source ethanol purely based on carbon stoichiometry is with $0.83 \mathrm{C}-\mathrm{mol} / \mathrm{C}$-mol as high as the IPP yield in E. coli. The overall stoichiometry for the formation of one mol IPP from ethanol (EtOH) is given by equation 8 .

$$
\begin{aligned}
& 3 \mathrm{EtOH}+9 \mathrm{ATP}+\mathrm{NADP}^{+}+3 \mathrm{NAD}^{+} \\
& =\mathrm{IPP}+\mathrm{CO}_{2}+9 \mathrm{ADP}+\mathrm{NADPH}+3 \mathrm{NADH}
\end{aligned}
$$

In contrast to glucose or glycerol as the carbon source, the formation of IPP from ethanol requires more energy but does not involve an additional carbon loss via $\mathrm{CO}_{2}$ (except the carbon loss in the terpenoid pathway itself). In fact, similarly to glycerol and E. coli, ethanol $(2,874,235 \mathrm{EMs})$ reveals an increased theoretical maximum product yield of $0.68 \mathrm{C}$ - $\mathrm{mol} / \mathrm{C}$-mol at zero growth as well as $0.59 \mathrm{C}$-mol/C-mol including biomass formation for the MVA pathway. The high number of elementary modes is again due to the activity of the glyoxylate cycle. The optimal flux distributions of yeast (without biomass formation) with only the MVA pathway reveal that the glycolysis, gluconeogenesis, pentose phosphate pathway and side product formation are not active. The flux passes pyruvate dehydrogenase bypass, glyoxylate cycle as well as citric acid cycle and respiratory chain to generate ATP. The energy deficiency preventing high IPP yields can be validated in silico by introducing artificial ATP or NADH sources which lead to the maximum yield of $0.83 \mathrm{C}-\mathrm{mol} / \mathrm{C}$-mol. As expected from these computations, the sesquiterpenoid yield of the same yeast strain was increased significantly in vivo by changing the carbon source from glucose $(0.0111 \mathrm{C}$-mol/C-mol $)$ to ethanol (0.1867 C-mol/C-mol) [8].
The introduction of the DXP pathway in silico and the growth on galactose, fructose or xylose lead to similar yields to those on glucose, except that the theoretical maximal IPP yields including biomass formation are slightly increased on xylose. With ethanol as substrate, the heterologous DXP pathway shows a lower potential compared to the native MVA pathway, with yields of $0.63 \mathrm{C}-\mathrm{mol} / \mathrm{C}$-mol at zero growth and $0.57 \mathrm{C}$-mol/C-mol including biomass formation. Lower yields are due to energy-consuming gluconeogenetic reactions which are essential to provide the precursors of the DXP pathway from ethanol. Nevertheless, the introduction of the DXP pathway could be beneficial for growth on glycerol. The theoretical maximum IPP yield is enhanced to $0.67 \mathrm{C}-\mathrm{mol} / \mathrm{C}-\mathrm{mol}$ at zero growth and $0.6 \mathrm{C}-\mathrm{mol} / \mathrm{C}-\mathrm{mol}$ including biomass formation. The cells would have a limitation in NADPH for high yield IPP production, which can be validated via the introduction of an artificial $\mathrm{NADPH}$ source leading to the maximum value of $0.83 \mathrm{C}$-mol/C-mol.

To summarize this subsection, sugars like glucose, galactose, fructose and even xylose lead to identical or similar theoretical maximal IPP yields in S. cerevisiae while the non-fermentable carbon sources ethanol and glycerol show the highest potential with respect to yield and network flexibility. Similarly, glycerol shows the highest potential in E. coli to supply IPP in high yields.

\section{Comparison of theoretical yields with experimental yields reported in literature}

A comparison of computed theoretical maximal IPP yields with terpenoid yields published in literature is shown in Table 2. The experimental carbon yields are still very low, even though strains used in these studies had been genetically modified within the respective terpenoid pathway. This highlights the tremendous potential of yield improvement. The terpenoid yield for yeast

Table 2 Comparison of theoretical maximal IPP yields with published terpenoid yields (given as C-mol/C-mol)

\begin{tabular}{lllcc}
\hline Organism & Pathway & Carbon source & $\begin{array}{c}\text { Maximum IPP yield } \\
\text { (this study) }\end{array}$ & $\begin{array}{c}\text { Experimental IPP yield estimated } \\
\text { from published yield }\end{array}$ \\
\hline E. coli & DXP & glycerol+ & 0.79 & $0.04[59]$ \\
E. coli & DXP & glycerol+ & 0.79 & $0.06[14]$ \\
E. coli & DXP + MVA & glycerol+ & 0.79 & $0.04[60]$ \\
S. cerevisiae & MVA & glucose+ & 0.53 & $0.003[61]$ \\
S. cerevisiae & MVA & glucose+ & 0.53 & $0.00006[62]$ \\
S. cerevisiae & MVA & galactose & 0.53 & $0.004[30]$ \\
S. cerevisiae & MVA & glucose & 0.53 & $0.0052[31]$ \\
S. cerevisiae & MVA & glucose & 0.53 & $0.01[8]$ \\
S. cerevisiae & MVA & glucose\&ethanol & $0.53 / 0.68$ & $0.06[8]$ \\
S. cerevisiae & MVA & ethanol & 0.68 & $0.19[8]$
\end{tabular}

Experimental yields of sesquiterpenoids are partly estimated and converted into IPP yields (only to that part of IPP that has been converted to the respective terpenoid); + indicates complex media. 
on ethanol was quite high in a study [8] indicating that the capacity of the terpene synthase or the terpenoid pathway (MVA) are not the only limiting factors but that the flux into these pathways is a limiting factor on other carbon sources like glucose. This shows that, besides the already very well-studied modifications within the terpenoid pathway, genetic modifications within the central carbon metabolism are indispensable to efficiently redirect the carbon flux to the terpenoid pathway ('push and pull' strategy).

\section{Target identification for metabolic engineering Identification of overexpression targets}

The identified limitations in the generation of sufficient energy and redox equivalents for high yield IPP production with both organisms can be used as starting point for metabolic engineering. Evidently, a reasonable approach would be to implement heterologous enzymes that are more efficient with respect to NAD(P)H or ATP consumption.

For E. coli, an increase in the maximum yield could be accomplished by the introduction of alternative pathways with less energy requirements or additional $\mathrm{NAD}(\mathrm{P}) \mathrm{H}$ sources. For instance, an additional heterologous $\mathrm{NADP}^{+}$-dependent glyceraldehyde-3-phosphate dehydrogenase enhances the theoretical maximum IPP yield to $0.71 \mathrm{C}-\mathrm{mol} / \mathrm{C}-\mathrm{mol}$ on glucose and to 0.83 $\mathrm{C}$-mol/C-mol on glycerol.

For $S$. cerevisiae, an increase in the maximum IPP yield could be accomplished by the introduction of alternative pathways with less energy requirements. S. cerevisiae possesses a mitochondrial pyruvate dehydrogenase complex and a cytosolic pyruvate dehydrogenase bypass to produce acetyl-CoA. Solely acetyl-CoA produced via the bypass, which requires ATP, can be used for terpenoid production since acetyl-CoA cannot be exported from the mitochondria. In contrast, $E$. coli possesses a cytosolic pyruvate dehydrogenase complex, which does not require energy in the form of ATP. In silico, a heterologous introduction of cytosolic pyruvate dehydrogenase complex from $E$. coli into $S$. cerevisiae leads to the theoretical maximum IPP yield of $0.56 \mathrm{C}-\mathrm{mol} /$ $\mathrm{C}$-mol and to a mode enabling biomass formation coupled to an increased IPP yield of $0.51 \mathrm{C}-\mathrm{mol} / \mathrm{C}-\mathrm{mol}$ on glucose. A cytosolic pyruvate dehydrogenase complex has to our knowledge not been introduced into yeast with the aim of enhancing terpenoid production. Though, a patent application indicates that the complex of E. coli $(\operatorname{lpdA}, \operatorname{ace} A$, aceF) might increase the cytosolic acetyl-CoA pool for butanol production [63].

A different approach would be to transfer the native MVA pathway from $S$. cerevisiae into the mitochondria. Likewise, on glucose, the theoretical maximum IPP yield at zero growth is enhanced to $0.56 \mathrm{C}-\mathrm{mol} / \mathrm{C}-\mathrm{mol}$ and the IPP yield including biomass formation is enhanced to $0.51 \mathrm{C}-\mathrm{mol} / \mathrm{C}-\mathrm{mol}$, if only the MVA in the mitochondria is active, or to 0.56 and $0.53 \mathrm{C}$-mol/C-mol if the MVA is active in the cytosol and the mitochondria. The mitochondrial IPP and DMAPP as well as the FPP pool have been harnessed successfully for terpenoid production [10]. However, the whole MVA pathway has not been transferred to the mitochondria. The advantage of this approach would be that the mitochondrial acetyl-CoA pool, which is formed via the pyruvate dehydrogenase complex that does not require ATP, would be used.

Alternatively, an ATP-citrate-lyase can be expressed heterologously in the cytosol. The enzyme forms cytosolic acetyl-CoA from citrate that has been exported from the mitochondria and thus circumvents pyruvate dehydrogenase bypass. The reaction is accompanied by the conversion of one mol ATP to ADP per acetyl-CoA, while the cytosolic pyruvate dehydrogenase bypass converts in the net one mol ATP to AMP. Thus, ATP-citrate-lyase is more energy-efficient and thus promising. The theoretical maximum IPP yield at zero growth is indeed enhanced to 0.55 $\mathrm{C}$-mol/C-mol. Moreover, a patent application indicates that this approach might be promising [64].

If the DXP pathway is introduced into yeast, a heterologous soluble transhydrogenase leads to a higher theoretical maximum IPP yield of $0.67 \mathrm{C}$-mol/C-mol on glucose. However, the introduction of a heterologous soluble transhydrogenase from Azotobacter vinelandii as well as the introduction of a membrane-bound enzyme from $E$. coli into $S$. cerevisiae led to a reduction of the NADPH pool including an increase in glycerol formation [65-67], which is the opposite of the desired effect. Though, the introduction of a heterologous $\mathrm{NADP}^{+}$dependent glyceraldehyde-3-phosphate dehydrogenase leads in silico to the same improvement in IPP yield on glucose and to a yield of $0.76 \mathrm{C}$-mol/C-mol on glycerol.

\section{Identification of knockout strategies}

The constrained minimal cut sets (cMCSs) approach allows the identification of all possible knockout combinations that eliminate undesired (low-yield) elementary modes and keep desired modes, which (i) have a specified minimum product yield and (ii) allow at least some biomass formation [40]. Hence, a selection pressure imposed by gene deletions forces the cell to produce a predefined minimum IPP yield to be able to grow. However, optimal biological functions are acquired through evolutionary processes. For in vivo validation, it has been shown that adaptive evolution is essential to improve production capabilities from sub-optimal metabolic states to optimal ones as predicted from in silico analysis [68,69].

In this study, cMCSs are used to identify combinations of gene deletions for both E. coli and S. cerevisiae 
to present new intervention targets for metabolic engineering.

cMCSs for E. coli Constrained minimal cut sets were computed for the E. coli wild type metabolic network on glucose $(36,590$ EMs) as well as glycerol as carbon source $(26,160$ elementary modes). For glucose, the deletion task was defined by collecting all elementary modes exhibiting an IPP yield lower than $0.25 \mathrm{C}-\mathrm{mol} / \mathrm{C}$-mol in the set of target modes $\tilde{\mathbf{T}}$ (33,087 EMs). The set of desired modes $\tilde{\mathbf{D}}$ comprises all EMs having (i) a minimum IPP yield of $0.25 \mathrm{C}$-mol/C-mol; and (ii) a simultaneous biomass yield necessarily higher than zero (268 modes) (see Figure 5). When at least one EM of the set of $\tilde{\mathbf{D}}$ should be preserved ( $n=1$; see Methods), a total of 488 cMCSs accomplishing the specified engineering goal were computed. Cut sets including diffusion processes or parts of respiratory chain were excluded from further considerations due to poor feasibility leaving $28 \mathrm{cMCSs}$ with two or four interventions (see Table 3). One group of cut sets forces a coupling of IPP production with biomass formation via redox equivalents. This is achieved by preventing oxygen uptake and thus respiration as well as side product formation and reactions within citric acid cycle that can reoxidize NADH (see Table 3). Hence, $\mathrm{NADH}$ has to be reoxidized via transhydrogenase which leads to excess NADPH production. Excess NADPH is then reoxidized via the DXP pathway. Applying these cut sets, the remaining elementary modes are anaerobic, exhibit a minimum IPP carbon yield of 0.38 and the maximum anaerobic IPP yield of 0.48 . However, the maximum possible biomass carbon yield is very low. A second group of cut sets includes the knockout of the lower part of glycolysis to force the cell to use the Entner-Doudoroff pathway instead, which results in less ATP but more NADPH. The cell cannot reoxidize excess NADPH by the additional knockout of transhydrogenase. Excess NADPH is then reoxidized via the DXP pathway. Remaining elementary modes are aerobic, exhibit a slightly lower minimum IPP carbon yield but have a much higher maximum biomass carbon yield than the anaerobic modes.

Another cut set included glucose-6-phosphate-isomerase, transhydrogenase, Entner-Doudoroff pathway as well as one specific part of respiratory chain. The cMCS is not shown in Table 3 due to poor feasibility of the last target. However, it serves as an example because it has been implemented. The coupling is again due to excess NADPH production. The knockout of glucose-6-phosphate-isomerase ( $\Delta p g i)$ has been done in E. coli in vivo and led to an increased flux through pentose phosphate pathway and thus to excess NADPH formation. However, an additional knockout of transhydrogenase ( $\Delta p g i$ $\triangle u d h A$ ) was lethal due to NADPH imbalance in this wild type strain [70]. This example illustrates that the principle of the method is effective but regulatory constraints may interfere with proposed knockout strategies. One can assume that the overexpression of a terpenoid synthase together with the DXP pathway as a sink and eventually adaptive evolution could balance the excess NADPH production via a 'push and pull' strategy and lead to the predicted coupling.

For glycerol, the deletion task was set up by (i) defining $\tilde{\mathbf{T}}$ as the set of elementary modes exhibiting an IPP yield of not more than $0.33 \mathrm{C}$-mol/C-mol (22,801 EMs);
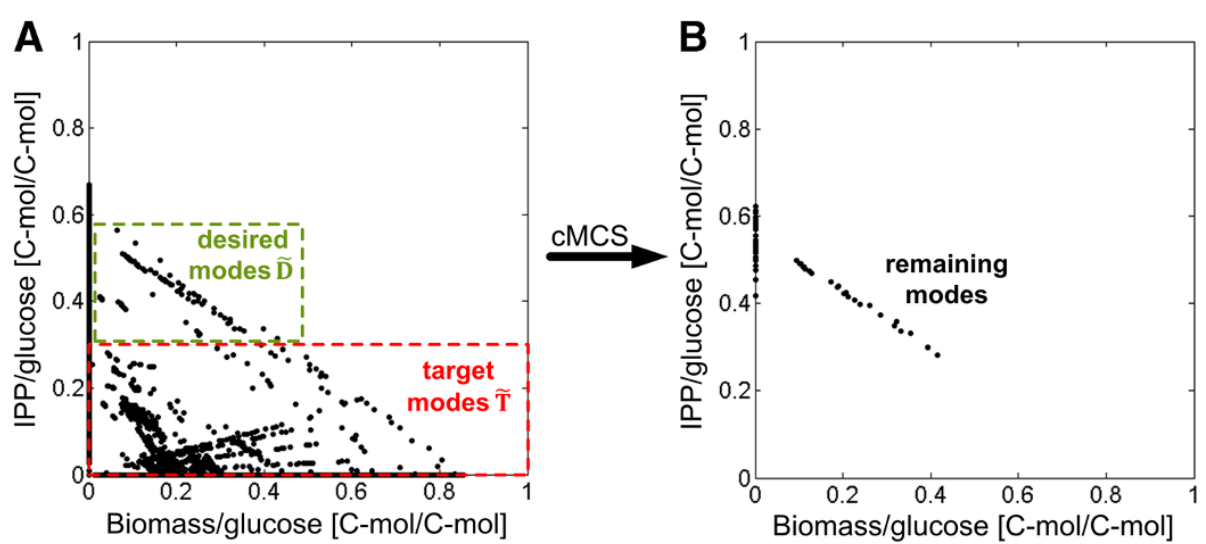

Figure 5 Concept of $\mathbf{c M C S}$ illustrated with an example of $\boldsymbol{E}$. coli. A: Solution space formed by the elementary modes of the wild type network of E. coli (36,590 EMs; compare Figure 2). The biomass yield on glucose is plotted against the IPP yield on glucose [C-mol/C-mol] of all computed elementary modes. The deletion task is defined by collecting all modes exhibiting an IPP yield lower than $0.25 \mathrm{C}-\mathrm{mol} / \mathrm{C}-\mathrm{mol}$ in the set of target modes $\tilde{\mathbf{T}}$ (33,087 EMs). The set of desired modes $\tilde{\mathbf{D}}$ comprises all EMs having (i) a minimum IPP yield of 0.25 C-mol/C-mol; and (ii) a simultaneous biomass yield strictly higher than zero (268 EMs). B: Example of one specific CMCS. The in silico knockout of transhydrogenase and phosphoglycerate mutase (strategy 2 in Table 3) leads to a reduction of elementary modes. Remaining elementary modes (191) display a minimum IPP yield of 0.28 and a maximum of 0.62 C-mol/C-mol as well as a maximum biomass yield of 0.41 C-mol/C-mol on glucose. In those modes, biomass formation is coupled to a minimum yield of IPP production. 
Table 3 cMCSs for $E$. coli: number of remaining elementary modes (EMs), minimum and maximum IPP yield as well as maximum biomass yield on glucose or glycerol [C-mol/C-mol] for different knockout strategies

\begin{tabular}{|c|c|c|c|c|}
\hline cMCS knockout strategy & EMs & $\begin{array}{l}\text { Min. IPP } \\
\text { yield }\end{array}$ & $\begin{array}{l}\text { Max. IPP } \\
\text { yield }\end{array}$ & $\begin{array}{l}\text { Max. biomass } \\
\text { yield }\end{array}$ \\
\hline Wild type on glucose & 36,590 & - & 0.67 & 0.85 \\
\hline $\begin{array}{l}\text { Strategy 1: } \mathrm{O}_{2} \text { uptake }+\{\text { lactate ex OR lactate formation }\}+\{\text { ethanol ex OR ethanol formation } O R \\
\text { acetaldehyde formation }+ \text { \{succinate ex OR succinate OR malate DH OR fumarate hydratase }\end{array}$ & $60-86$ & 0.38 & 0.48 & 0.08 \\
\hline Strategy 2: transhydrogenase + \{phosphoglycerate mutase OR enolase $\}$ & 191 & 0.28 & 0.62 & 0.41 \\
\hline Strategy 3: transhydrogenase $+\{$ GAP DH OR phosphoglycerate kinase $\}$ & 191 & 0.35 & 0.62 & 0.32 \\
\hline Wild type on glycerol & 26,160 & - & 0.78 & 0.99 \\
\hline $\begin{array}{l}\text { Strategy 1: transhydrogenase + pyruvate kinase }+ \text { pyruvate DH complex + ribulose-phosphate } \\
\text { 3-epimerase + acetate formation + glyoxylate shunt }+\{\text { succinate DH OR fumarate hydratase }\}\end{array}$ & 22 & 0.38 & 0.68 & 0.40 \\
\hline
\end{tabular}

$\mathrm{DH}=$ dehydrogenase; ex = excretion.

(ii) defining $\tilde{\mathbf{D}}$ as all EMs with a minimum IPP yield of $0.33 \mathrm{C}-\mathrm{mol} / \mathrm{C}$-mol and a simultaneous biomass formation strictly higher than zero (570 EMs); and (iii) demanding that at least one EM of $\tilde{\mathbf{D}}$ is preserved $(n=1)$. A total of 769 cMCSs were computed, while cut sets that cannot be realized in practice (including diffusion processes, parts of respiratory chain, ATP maintenance etc.) were excluded from further considerations leaving only $8 \mathrm{cMCSs}$ with eight interventions each (see Table 3). Feasible minimal cut sets include the knockout of transhydrogenase, pyruvate kinase, pyruvate dehydrogenase complex, ribulosephosphate 3-epimerase, acetate formation, glyoxylate shunt and citric acid cycle (succinate dehydrogenase or fumarate hydratase). A minimum IPP carbon yield of 0.38 $\mathrm{C}$-mol/C-mol can be guaranteed but only two of the remaining elementary modes enable biomass formation.

The presented minimal cut set strategies can also be applied to E. coli strains carrying the additional MVA pathway. The IPP and biomass yields of remaining elementary modes are only slightly modified (results not shown).

The cMCSs approach points to new and promising combinations of metabolic engineering targets. The predicted minimal IPP yields are much higher than the yields that have been published in literature so far. Nevertheless, kinetics and regulation, especially of the DXP pathway, may impose additional challenges to be solved in order to achieve high terpenoid yields with $E$. coli.

cMCSs for S. cerevisiae Constrained minimal cut sets were computed based on the wild type metabolic network with glucose as carbon source (9,844 EMs). Modes exhibiting an IPP yield of not more than 0.25 $\mathrm{C}$-mol/C-mol were collected in the set of target modes $\tilde{\mathbf{T}}$ (9,453 EMs). All EMs exhibiting (i) an IPP yield of not less than $0.25 \mathrm{C}$-mol/C-mol; and (ii) a concurrent biomass yield strictly higher than zero were defined as desired modes $\tilde{\mathbf{D}}$ (322 EMs). While preserving at least one of the desired modes $(n=1)$, a total of 407 cMCSs were computed. Cut sets including mitochondrial transport systems, diffusion, ATP maintenance etc. were excluded from further considerations leaving 8 feasible cMCSs with three to six interventions (Table 4). All remaining cMCS require blocking of acetate secretion and either of ethanol secretion or of all alcohol dehydrogenases (cytosolic and mitochondrial). The prevention of acetate and ethanol production is crucial as the engineering goal cannot be accomplished without those two targets. An additional target is the partial knockout of citric acid cycle after $\alpha$-ketoglutarate, namely $\alpha$-ketoglutarate dehydrogenase or succinyl-CoA

Table 4 cMCSs for S. cerevisiae: number of remaining elementary modes (EMs), minimum and maximum IPP carbon yield as well as maximum biomass carbon yield on glucose [C-mol/C-mol] for different knockout strategies

\begin{tabular}{|c|c|c|c|c|}
\hline cMCS knockout strategy & EMs & $\begin{array}{l}\text { Min. IPP } \\
\text { yield }\end{array}$ & $\begin{array}{l}\text { Max. IPP } \\
\text { yield }\end{array}$ & $\begin{array}{l}\text { Max. biomass } \\
\text { yield }\end{array}$ \\
\hline Wild type & 9,844 & - & 0.53 & 0.80 \\
\hline Strategy 1: acetate ex $+\{$ ethanol ex OR alcohol DH $\}+\{$ a-ketoglutarate $\mathrm{DH}$ OR succinyl-CoA ligase $\}$ & $142 / 48$ & 0.33 & 0.53 & 0.29 \\
\hline Strategy 2: acetate ex $+\{$ ethanol ex $\mathrm{OR}$ alcohol $\mathrm{DH}\}+$ malate $\mathrm{DH}+$ malic enzyme & $60 / 21$ & 0.33 & 0.53 & 0.29 \\
\hline $\begin{array}{l}\text { Strategy 3: acetate ex }+\{\text { ethanol ex } \mathrm{OR} \text { alcohol } \mathrm{DH}\}+\text { malate } \mathrm{DH}+\mathrm{NAD}^{+} \text {-dependent isocitrate } \\
\mathrm{DH}+\text { pyruvate } \mathrm{DH} \text { complex }\end{array}$ & $51 / 19$ & 0.27 & 0.53 & 0.37 \\
\hline Wild type plus DXP pathway & 11,738 & - & 0.64 & 0.80 \\
\hline Strategy 1: glucose-6-phosphate-isomerase & 631 & 0.37 & 0.63 & 0.35 \\
\hline
\end{tabular}


ligase (succinate dehydrogenase or fumarate hydratase would be an alternative as well but were not considered due to possible succinate secretion). Alternatively malate dehydrogenase and malic enzyme can be deleted for a partial knockout of citric acid cycle. A second alternative is malate dehydrogenase, $\mathrm{NAD}^{+}$dependent isocitrate dehydrogenase as well as pyruvate dehydrogenase complex.

Possible knockout combinations were simulated and Table 4 shows the number of remaining elementary modes and the minimal and maximal IPP and biomass carbon yields when applying the respective cMCS. All minimal cut sets lead to a reduced maximum biomass yield and a reduced number of elementary modes. However, a certain flexibility is preserved. The IPP carbon yield can reach the maximum value of the wild type, and a minimum value of 0.27 or 0.33 is computed. Due to the partial disruption of citric acid cycle the cell is not able to convert glucose completely to $\mathrm{CO}_{2}$. Hence, the cell is forced to secrete products like ethanol or acetate. The repression of acetate and ethanol excretion forces the cell to find another sink like IPP. Targets identified for glucose can also be applicable to other sugars like galactose or fructose.

The knockout of succinyl-CoA ligase $(\triangle L S C 1)$ was implemented in S. cerevisiae and an enhanced acetate secretion was observed [71]. This indicates that the principle of the method is effective even though acetate is produced instead of channeling the flux to acetyl-CoA, the precursor of the MVA pathway. Nevertheless, acetate secretion can be lowered by the overexpression of acetyl-CoA synthetase [48]. Moreover, an overexpression of the MVA pathway together with a terpenoid synthase might channel the flux to IPP via the 'push and pull' strategy.

cMCSs including a heterologous DXP pathway are basically identical to those of the wild type. Thus, presented strategies can also be applied to a strain carrying an additional DXP pathway if the pathway was active in vivo. However, the minimal IPP yields are unchanged and thus the heterologous DXP pathway would be of no benefit. Nevertheless, one additional interesting intervention strategy was computed. The single knockout of glucose-6-phosphate-isomerase leads to a coupling of biomass formation to a minimal IPP yield of $0.37 \mathrm{C}-\mathrm{mol} /$ $\mathrm{C}$-mol. The knockout of glucose-6-phosphate-isomerase forces the cell to direct the flux completely to pentose phosphate pathway, which leads to excess NADPH production. Wild type yeast cells are not able to compensate excess NADPH as they do not possess a transhydrogenase. Further, NADH dehydrogenases (Ndilp, Nde1p, Nde2p) do not oxidize NADPH and malic enzyme reaction seems to be irreversible in vivo [72,73]. Yeast cells are not able to grow on glucose after the knockout of PGI1 gene encoding glucose-6-phosphate-isomerase [74] and no elementary modes could be computed for the wild type network including only the MVA pathway with the single knockout of glucose-6-phosphate-isomerase. Nevertheless, an active DXP pathway could serve as a NADPH sink as it requires more NADPH and less ATP per mol IPP than the MVA pathway.

Minimal cut sets were also computed for the metabolic network with xylose as carbon source including either the XI or the XR-XDH pathway together with the MVA pathway. Identified knockout targets as well as yields after the implementation of a minimal cut set for the XI pathway are identical to those of glucose. Knockout targets for the XR-XDH pathway are different. Minimal cut sets only exist with an additional target, ribulose-5-phosphate-3-epimerase in pentose phosphate pathway. Cut sets including pyruvate dehydrogenase complex do not exist. Moreover, possible IPP yields after the implementation of a minimal cut set are slightly different and can vary between 0.30 and $0.50 \mathrm{C}$-mol/C-mol.

To our knowledge, a disruption of citric acid cycle has not been done with the aim to enhance terpenoid production. Predicted minimal IPP yields are much higher than yields that have been achieved in literature so far. Thus, targets identified by the minimal cut sets approach are completely new and promising for an enhanced terpenoid yield on sugars with $S$. cerevisiae. Moreover, the knockout of glucose-6-phosphate-isomerase appears as a very promising strategy to enhance terpenoid production if the DXP pathway can be functionally expressed in yeast.

\section{Conclusions}

The use and optimization of microorganisms for an ecologically and economically efficient production of plant terpenoids is a contemporary issue in academic research as well as industry. The most widely used model organisms $E$. coli and S. cerevisiae as well as their respective terpenoid pathway, heterologous enzymes and different carbon sources have been analyzed in silico in this study with respect to their potential as terpenoid factories. The DXP pathway of E. coli has a tremendously higher potential for high IPP yields (nearly 50\% higher) than the MVA pathway of yeast with respect to carbon stoichiometry due to their different precursors. However, kinetics, regulation and a balanced overexpression have to be considered for in vivo production. Further research should focus on the optimization of the DXP pathway to tap its full potential. E. coli wild type shows a higher theoretical potential to supply IPP in high yields than S. cerevisiae wild type. However, the theoretical maximal yields can be enhanced in both organisms by introducing heterologous enzymes or whole pathways or by simply changing the carbon source. Moreover, new constrained minimal cut set strategies can be applied to both hosts 
to enforce a coupling of growth to a minimum IPP yield which is higher than any yield published in scientific literature so far.

\section{Methods}

\section{Elementary modes and minimal cut sets}

Consider a given metabolic reaction network with $\mathrm{q} \times \mathrm{p}$ stoichiometric matrix $\mathbf{N}$ (with q reactions and p metabolites) and a set Irrev of irreversible reactions. The set of steady-state flux vectors $\mathbf{r}$ form the convex (flux) cone reads:

$$
\boldsymbol{F}=\left\{\mathbf{r} \in \mathfrak{R}^{q} \mid \mathbf{N r}=\mathbf{0}, \quad r_{i} \geq 0 \forall i \in \text { Irrev }\right\}
$$

An Elementary Mode is a non-decomposable vector $\mathbf{e}$ $\in \boldsymbol{F}$, i.e. for every vector $\mathbf{r} \in \boldsymbol{F}, \mathbf{r} \neq 0$, one has $\operatorname{supp}(\mathbf{e}) \subseteq$ $\operatorname{supp}(\mathbf{r})$, where the support of a vector is defined as the index set of all reactions carrying a non-zero flux $(\operatorname{supp}(\mathbf{r})=$ $\left.\left\{i \mid r_{i} \neq 0\right\}\right)$. EMs correspond to minimal functional units (pathways or cycles) of a metabolic network and are useful to study various functional network properties $[34,36]$.

In contrast, a minimal cut set (MCS) is a (support-) minimal set $C$ of reactions, the removal of which will block (disrupt) a given set $\boldsymbol{M}$ of target flux vectors, $\boldsymbol{M} \subseteq \boldsymbol{F}[75,76]:$

$$
\left\{\mathbf{r} \in \boldsymbol{F} \mid r_{i}=0, \forall i \in \boldsymbol{C}\right\} \cap \boldsymbol{M}=\varnothing
$$

One drawback of the original definition of MCSs is that besides the targeted functionalities some desired functionalities can be lost as well. Therefore, a generalization to constrained MCSs was introduced [40] which can be described as follows (in the following, EMs are represented by their support, i.e. by the set of reactions carrying a non-zero flux in the EM):

1) Define a set of target modes $\tilde{\mathbf{T}}$ (where each target mode $\mathrm{t}$ is represented by its support $\boldsymbol{T}=\operatorname{supp}(\mathbf{t})$ ).

2) Define a set of desired modes $\tilde{\mathbf{D}}$ (where each desired mode $\mathbf{d}$ is represented by its support $\boldsymbol{D}=\operatorname{supp}(\mathbf{d})$ ).

3) Specify a number $n$ quantifying the minimal number of EMs from $\tilde{\mathbf{D}}$ that must be kept in the network, i.e. a cMCS $\boldsymbol{C}$ fulfills: $\forall \boldsymbol{T} \in \tilde{\mathbf{T}}: \boldsymbol{C} \cap \boldsymbol{T} \neq \varnothing \wedge|\{\boldsymbol{D} \in \tilde{\mathbf{D}} \mid \boldsymbol{D} \cap \boldsymbol{C}=\varnothing\}| \geq n$.

cMCSs provide a flexible and convenient approach for formulating and solving intervention problems. The approach also allows one to compute interventions that lead to robust coupling of product and biomass formation (coupling even for suboptimal growth). To compute the cMCSs, we applied an adapted Berge algorithm (allowing the computation of cMCSs as minimal hitting sets of $\tilde{\mathbf{T}}$ subject to $\tilde{\mathbf{D}}$ and $n$ ), implemented in the software package CellNetAnalyzer [77].

\section{Metabolic networks}

A comprehensive analysis of the complete set of elementary modes is still infeasible with genome-scale metabolic models. Therefore, stoichiometric models of the central metabolism of E. coli and S. cerevisiae were built up manually. The basic glucose network for $E$. coli consists of 65 reactions, of which 21 are reversible, and 50 metabolites (12 external). For $S$. cerevisiae the basic glucose network comprises 69 reactions, thereof 30 reversible, and 60 metabolites (8 external). The P/O-ratios (mol ATP produced per $0.5 \mathrm{~mol} \mathrm{O}_{2}$ reduced) representing the efficiency of ATP production are assumed to be 1.2 for $S$. cerevisiae [78,79] and for E. coli 2 from NADH and 1 from quinol $[39,43,80]$. The metabolic networks of $S$. cerevisiae and $E$. coli have been constructed considering the current knowledge from genome-scale models and literature data [81-86]. The most important reactions are depicted in Figures 3 and 4, respectively. Details of the models (including the complete list of enzymes, genes and considered literature) are given in Additional file 1.

\section{Additional file}

Additional file 1: Metabolic networks. Metabolic networks of S. cerevisiae and E. coli including the complete list of genes, heterologous enzymes, alternative carbon sources and considered literature.

\begin{abstract}
Abbreviations
AcCoA: Acetyl-CoA; ACL: ATP-citrate-lyase; CDW: Cell dry weight; CMCS: Constrained minimal cut set; DH: Dehydrogenase; DMAPP: Dimethylallyl diphosphate; DXP: 1-deoxy-D-xylulose 5-phosphate; EM: Elementary mode; EMA: Elementary mode analysis; EtOH: Ethanol; Ex: Excretion; FPP: Farnesyl diphosphate; GAP: Glyceraldehyde-3-phosphate; GLC: Glucose; GLYC: Glycerol; IPP: Isopentenyl diphosphate; MCS: Minimal cut set; MEP: 2C-methyl-D-erythriol 4-phosphate; MVA: Mevalonate;

PDH: Cytosolic pyruvate dehydrogenase complex; PYR: Pyruvate; TH: Soluble and energy-independent transhydrogenase; $X I$ : Xylose isomerase; XR$\mathrm{XDH}$ : Xylose reductase and xylitol dehydrogenase; XYL: Xylose.
\end{abstract}

\section{Competing interests}

The authors declare that they have no competing interests.

\section{Authors' contributions}

EG did all the computations and interpretations. OH and SK supported the constrained minimal cut sets computations. EG and VS designed the study. EG, VS, OH and SK wrote and drafted the manuscript. OK supervised the research. VS conceived of the study and supervised the research. All authors read and approved the final manuscript.

\section{Acknowledgements}

This work was funded by the Ministry of Innovation, Science and Research of the German Federal State of North Rhine-Westphalia (NRW) and by TU Dortmund through a scholarship to EG from the CLIB-Graduate Cluster Industrial Biotechnology (CLIB ${ }^{2021}$ ). SK and $\mathrm{OH}$ thank for financial support by the Ministry of Education and Research of Saxony-Anhalt ('Research Center Dynamic Systems: Biosystems Engineering').

\section{Author details}

${ }^{1}$ Technical Biochemistry, Department of Biochemical and Chemical Engineering, TU Dortmund University, Emil-Figge-Str. 66, 44227 Dortmund, Germany. ${ }^{2}$ Analysis and Redesign of Biological Networks, Max Planck Institute for Dynamics of Complex Technical Systems, Sandtorstr. 1, 39106 Magdeburg, Germany. ${ }^{3}$ Biomax Informatics AG, Robert-Koch-Str. 2, 82152 Planegg, Germany. 
Received: 2 July 2013 Accepted: 15 September 2013

Published: 23 September 2013

\section{References}

1. Hong KK, Nielsen J: Metabolic engineering of Saccharomyces cerevisiae: a key cell factory platform for future biorefineries. Cell Mol Life Sci 2012, 69:2671-2690

2. Kirby J, Keasling JD: Biosynthesis of plant isoprenoids: perspectives for microbial engineering. Annu Rev Plant Biol 2009, 60:335-355.

3. Woodrow CJ, Haynes RK, Krishna S: Artemisinins. Postgrad Med J 2005, 81:71-78.

4. Davies HM: Organic chemistry: Synthetic lessons from nature. Nature 2009, 459:786-787.

5. Jiang M, Stephanopoulos G, Pfeifer BA: Toward biosynthetic design and implementation of Escherichia coli-derived paclitaxel and other heterologous polyisoprene compounds. App/ Environ Microbiol 2012, 78:2497-2504

6. Kirby J, Keasling JD: Metabolic engineering of microorganisms for isoprenoid production. Nat Prod Rep 2008, 25:656-661.

7. Ro DK, Paradise EM, Ouellet M, Fisher KJ, Newman KL, Ndungu JM, Ho KA, Eachus RA, Ham TS, Kirby J, et al: Production of the antimalarial drug precursor artemisinic acid in engineered yeast. Nature 2006, 440:940-943.

8. Westfall PJ, Pitera DJ, Lenihan JR, Eng D, Woolard FX, Regentin R, Horning T, Tsuruta H, Melis DJ, Owens A, et al: Production of amorphadiene in yeast, and its conversion to dihydroartemisinic acid, precursor to the antimalarial agent artemisinin. Proc Natl Acad Sci U S A 2012, 109:E111-E118.

9. Chang MC, Eachus RA, Trieu W, Ro DK, Keasling JD: Engineering Escherichia coli for production of functionalized terpenoids using plant P450s. Nat Chem Biol 2007, 3:274-277.

10. Farhi M, Marhevka E, Masci T, Marcos E, Eyal Y, Ovadis M, Abeliovich H, Vainstein A: Harnessing yeast subcellular compartments for the production of plant terpenoids. Metab Eng 2011, 13:474-481.

11. Kampranis SC, Makris AM: Developing a yeast cell factory for the production of terpenoids. Comput Struct Biotechnol J 2012, 3:1-7.

12. Maury J, Asadollahi MA, Moller K, Clark A, Nielsen J: Microbial isoprenoid production: an example of green chemistry through metabolic engineering. Adv Biochem Eng Biotechnol 2005, 100:19-51.

13. Partow S, Siewers V, Daviet L, Schalk M, Nielsen J: Reconstruction and evaluation of the synthetic bacterial MEP Pathway in Saccharomyces cerevisiae. PLoS One 2012, 7:e52498.

14. Ajikumar PK, Xiao WH, Tyo KE, Wang Y, Simeon F, Leonard E, Mucha O, Phon TH, Pfeifer B, Stephanopoulos G: Isoprenoid pathway optimization for taxol precursor overproduction in Escherichia coli. Science 2010, 330:70-74.

15. Huang Q, Roessner CA, Croteau R, Scott Al: Engineering Escherichia coli for the synthesis of taxadiene, a key intermediate in the biosynthesis of taxol. Bioorg Med Chem 2001, 9:2237-2242.

16. Kim SW, Keasling JD: Metabolic engineering of the nonmevalonate isopentenyl diphosphate synthesis pathway in Escherichia coli enhances lycopene production. Biotechnol Bioeng 2001, 72:408-415.

17. Martin VJ, Pitera DJ, Withers ST, Newman JD, Keasling JD: Engineering a mevalonate pathway in Escherichia coli for production of terpenoids. Nat Biotechnol 2003, 21:796-802.

18. Morrone D, Lowry L, Determan MK, Hershey DM, Xu M, Peters RJ: Increasing diterpene yield with a modular metabolic engineering system in E. coli: comparison of MEV and MEP isoprenoid precursor pathway engineering. Appl Microbiol Biotechnol 2010, 85:1893-1906.

19. Rodriguez-Villalon A, Perez-Gil J, Rodriguez-Concepcion M: Carotenoid accumulation in bacteria with enhanced supply of isoprenoid precursors by upregulation of exogenous or endogenous pathways. J Biotechnol 2008, 135:78-84

20. Yuan LZ, Rouviere PE, Larossa RA, Suh W: Chromosomal promoter replacement of the isoprenoid pathway for enhancing carotenoid production in E. coli. Metab Eng 2006, 8:79-90.

21. Zhou K, Zhou L, Lim Q, Zou R, Stephanopoulos G, Too HP: Novel reference genes for quantifying transcriptional responses of Escherichia coli to protein overexpression by quantitative PCR. BMC Mol Biol 2011, 12:18.

22. Kizer $L$, Pitera DJ, Pfleger BF, Keasling JD: Application of functional genomics to pathway optimization for increased isoprenoid production Appl Environ Microbiol 2008, 74:3229-3241.
23. Pitera DJ, Paddon CJ, Newman JD, Keasling JD: Balancing a heterologous mevalonate pathway for improved isoprenoid production in Escherichia coli. Metab Eng 2007, 9:193-207.

24. Tsuruta H, Paddon CJ, Eng D, Lenihan JR, Horning T, Anthony LC, Regentin $R$, Keasling JD, Renninger NS, Newman JD: High-level production of amorpha-4,11-diene, a precursor of the antimalarial agent artemisinin, in Escherichia coli. PLoS One 2009, 4:e4489.

25. Yoon SH, Lee SH, Das A, Ryu HK, Jang HJ, Kim JY, Oh DK, Keasling JD, Kim SW: Combinatorial expression of bacterial whole mevalonate pathway for the production of beta-carotene in E. coli. J Biotechnol 2009, 140:218-226.

26. Polakowski T, Stahl U, Lang C: Overexpression of a cytosolic hydroxymethylglutaryl-CoA reductase leads to squalene accumulation in yeast. Appl Microbiol Biotechnol 1998, 49:66-71.

27. Donald KA, Hampton RY, Fritz IB: Effects of overproduction of the catalytic domain of 3-hydroxy-3-methylglutaryl coenzyme a reductase on squalene synthesis in Saccharomyces cerevisiae. Appl Environ Microbiol 1997, 63:3341-3344.

28. Gardner RG, Hampton RY: A highly conserved signal controls degradation of 3-hydroxy-3-methylglutaryl-coenzyme a (HMG-CoA) reductase in eukaryotes. J Biol Chem 1999, 274:31671-31678.

29. Paradise EM, Kirby J, Chan R, Keasling JD: Redirection of flux through the FPP branch-point in Saccharomyces cerevisiae by down-regulating squalene synthase. Biotechnol Bioeng 2008, 100:371-378.

30. Asadollahi MA, Maury J, Moller K, Nielsen KF, Schalk M, Clark A, Nielsen J: Production of plant sesquiterpenes in Saccharomyces cerevisiae: effect of ERG9 repression on sesquiterpene biosynthesis. Biotechnol Bioeng 2008, 99:666-677.

31. Scalcinati G, Partow S, Siewers V, Schalk M, Daviet L, Nielsen J: Combined metabolic engineering of precursor and co-factor supply to increase alpha-santalene production by Saccharomyces cerevisiae. Microb Cell Fact 2012, 11:117.

32. Melzer G, Esfandabadi ME, Franco-Lara E, Wittmann C: Flux Design: In silico design of cell factories based on correlation of pathway fluxes to desired properties. BMC Syst Biol 2009, 3:120.

33. Papin JA, Stelling J, Price ND, Klamt S, Schuster S, Palsson BO: Comparison of network-based pathway analysis methods. Trends Biotechnol 2004, 22:400-405.

34. Schuster S, Fell DA, Dandekar T: A general definition of metabolic pathways useful for systematic organization and analysis of complex metabolic networks. Nat Biotechnol 2000, 18:326-332.

35. Schuster S, Hilgetag C, Woods JH, Fell DA: Reaction routes in biochemical reaction systems: algebraic properties, validated calculation procedure and example from nucleotide metabolism. J Math Biol 2002, 45:153-181.

36. Trinh CT, Wlaschin A, Srienc F: Elementary mode analysis: a useful metabolic pathway analysis tool for characterizing cellular metabolism. Appl Microbiol Biotechnol 2009, 81:813-826.

37. Krömer JO, Wittmann C, Schröder H, Heinzle E: Metabolic pathway analysis for rational design of L-methionine production by Escherichia coli and Corynebacterium glutamicum. Metab Eng 2006, 8:353-369.

38. Carlson R, Fell D, Srienc F: Metabolic pathway analysis of a recombinant yeast for rational strain development. Biotechnol Bioeng 2002, 79:121-134.

39. Carlson R, Srienc F: Fundamental Escherichia coli biochemical pathways for biomass and energy production: identification of reactions. Biotechnol Bioeng 2004, 85:1-19.

40. Hädicke O, Klamt S: Computing complex metabolic intervention strategies using constrained minimal cut sets. Metab Eng 2011, 13:204-213.

41. Unrean $P$, Trinh $C T$, Srienc F: Rational design and construction of an efficient $E$. coli for production of diapolycopendioic acid. Metab Eng 2010, 12:112-122.

42. Trinh CT, Carlson R, Wlaschin A, Srienc F: Design, construction and performance of the most efficient biomass producing $E$. coli bacterium. Metab Eng 2006, 8:628-638.

43. Trinh CT, Unrean P, Srienc F: Minimal Escherichia coli cell for the most efficient production of ethanol from hexoses and pentoses. App/ Environ Microbiol 2008, 74:3634-3643.

44. Trinh CT, Srienc F: Metabolic engineering of Escherichia coli for efficient conversion of glycerol to ethanol. Appl Environ Microbiol 2009, 75:6696-6705.

45. Alper H, Miyaoku K, Stephanopoulos G: Construction of lycopeneoverproducing $E$. coli strains by combining systematic and combinatorial gene knockout targets. Nat Biotechnol 2005, 23:612-616. 
46. Asadollahi MA, Maury J, Patil KR, Schalk M, Clark A, Nielsen J: Enhancing sesquiterpene production in Saccharomyces cerevisiae through in silico driven metabolic engineering. Metab Eng 2009, 11:328-334.

47. Choi HS, Lee SY, Kim TY, Woo HM: In silico identification of gene amplification targets for improvement of lycopene production. Appl Environ Microbiol 2010, 76:3097-3105

48. Shiba Y, Paradise EM, Kirby J, Ro DK, Keasling JD: Engineering of the pyruvate dehydrogenase bypass in Saccharomyces cerevisiae for highlevel production of isoprenoids. Metab Eng 2007, 9:160-168.

49. Farmer WR, Liao JC: Precursor balancing for metabolic engineering of lycopene production in Escherichia coli. Biotechnol Prog 2001, 17:57-61.

50. Zhao J, Li Q, Sun T, Zhu X, Xu H, Tang J, Zhang X, Ma Y: Engineering central metabolic modules of Escherichia coli for improving $\beta$-carotene production. Metab Eng 2013, 17:42-50.

51. Navarro-Avino JP, Prasad R, Miralles VJ, Benito RM, Serrano R: A proposal for nomenclature of aldehyde dehydrogenases in Saccharomyces cerevisiae and characterization of the stress-inducible ALD2 and ALD3 genes. Yeast 1999, 15:829-842.

52. Carlsen S, Ajikumar PK, Formenti LR, Zhou K, Phon TH, Nielsen ML, Lantz AE, Kielland-Brandt MC, Stephanopoulos G: Heterologous expression and characterization of bacterial 2-C-methyl-D-erythritol-4-phosphate pathway in Saccharomyces cerevisiae. Appl Microbiol Biotechnol 2013, 97:5753-5769.

53. Dobson R, Gray V, Rumbold K: Microbial utilization of crude glycerol for the production of value-added products. J Ind Microbiol Biotechnol 2012, 39:217-226

54. Randez-Gil F, Sanz P, Prieto JA: Engineering baker's yeast: room for improvement. Trends Biotechnol 1999, 17:237-244.

55. Carvalheiro F, Duarte LC, Gírio FM: Hemicellulose biorefineries: a review on biomass pretreatments. J Sci Ind Res 2008, 67:849-864.

56. Pandey A, Soccol CR, Nigam P, Soccol VT: Biotechnological potential of agro-industrial residues: I: sugarcane bagasse. Bioresour Technol 2000, 74:69-80.

57. da Silva GP, Mack M, Contiero J: Glycerol: a promising and abundant carbon source for industrial microbiology. Biotechnol Adv 2009, 27:30-39.

58. Karhumaa K, Garcia Sanchez R, Hahn-Hagerdal B, Gorwa-Grauslund MF: Comparison of the xylose reductase-xylitol dehydrogenase and the xylose isomerase pathways for xylose fermentation by recombinant Saccharomyces cerevisiae. Microb Cell Fact 2007, 6:5.

59. Meng $H$, Wang $Y$, Hua $Q$, Zhang $S$, Wang $X$ : In silico analysis and experimental improvement of taxadiene heterologous biosynthesis in Escherichia coli. Biotechnol Bioprocess Eng 2011, 16:205-215.

60. Wang C, Yoon SH, Shah AA, Chung YR, Kim JY, Choi ES, Keasling JD, Kim SW: Farnesol production from Escherichia coli by harnessing the exogenous mevalonate pathway. Biotechnol Bioeng 2010, 107:421-429.

61. Muramatsu M, Ohto C, Obata S, Sakuradani E, Shimizu S: Alkaline pH enhances farnesol production by Saccharomyces cerevisiae. J Biosci Bioeng 2009, 108:52-55

62. Lindahl AL, Olsson ME, Mercke P, Tollbom O, Schelin J, Brodelius M, Brodelius PE: Production of the artemisinin precursor amorpha-4,11-diene by engineered Saccharomyces cerevisiae. Biotechnol Lett 2006, 28:571-580.

63. Gunawardena U, Meinhold P, Peters MW, Urano RM, Feldmann RMR: Butanol production by metabolically engineered yeast. 2010. US 2010/0062505 A1.

64. Hansen J: Method of producing isoprenoid compounds in yeast. 2013. US 2013/0137138 A1.

65. Nissen TL, Anderlund M, Nielsen J, Villadsen J, Kielland-Brandt MC: Expression of a cytoplasmic transhydrogenase in Saccharomyces cerevisiae results in formation of 2-oxoglutarate due to depletion of the NADPH pool. Yeast 2001, 18:19-32.

66. Jeun Y-S, Kim M-D, Park Y-C, Lee T-H, Yoo M-S, Ryu Y-W, Seo J-H: Expression of Azotobacter vinelandii soluble transhydrogenase perturbs xylose reductasemediated conversion of xylose to xylitol by recombinant Saccharomyces cerevisiae. J Mol Cata B: Enzym 2003, 26:251-256.

67. Anderlund M, Nissen TL, Nielsen J, Villadsen J, Rydstrom J, Hahn-Hagerdal B, Kielland-Brandt MC: Expression of the Escherichia coli pntA and pntB genes, encoding nicotinamide nucleotide transhydrogenase, in Saccharomyces cerevisiae and its effect on product formation during anaerobic glucose fermentation. Appl Environ Microbiol 1999, 65:2333-2340.

68. Fong SS, Burgard AP, Herring CD, Knight EM, Blattner FR, Maranas CD, Palsson BO: In silico design and adaptive evolution of Escherichia coli for production of lactic acid. Biotechnol Bioeng 2005, 91:643-648.
69. Ibarra RU, Edwards JS, Palsson BO: Escherichia coli K-12 undergoes adaptive evolution to achieve in silico predicted optimal growth. Nature 2002, 420:186-189.

70. Sauer U, Canonaco F, Heri S, Perrenoud A, Fischer E: The soluble and membrane-bound transhydrogenases UdhA and PntAB have divergent functions in NADPH metabolism of Escherichia coli. J Biol Chem 2004, 279:6613-6619.

71. Blank LM, Kuepfer L, Sauer U: Large-scale 13C-flux analysis reveals mechanistic principles of metabolic network robustness to null mutations in yeast. Genome Biol 2005, 6:R49.

72. Luttik MA, Overkamp KM, Kotter P, de Vries S, van Dijken JP, Pronk JT: The Saccharomyces cerevisiae NDE1 and NDE2 genes encode separate mitochondrial NADH dehydrogenases catalyzing the oxidation of cytosolic NADH. J Biol Chem 1998, 273:24529-24534.

73. Boles $\mathrm{E}$, de Jong-Gubbels P, Pronk JT: Identification and characterization of MAE1, the Saccharomyces cerevisiae structural gene encoding mitochondrial malic enzyme. J Bacteriol 1998, 180:2875-2882.

74. Aguilera A: Deletion of the phosphoglucose isomerase structural gene makes growth and sporulation glucose dependent in Saccharomyces cerevisiae. Mol Gen Genet 1986, 204:310-316.

75. Klamt S, Gilles ED: Minimal cut sets in biochemical reaction networks. Bioinformatics 2004, 20:226-234.

76. Klamt S: Generalized concept of minimal cut sets in biochemical networks. Biosystems 2006, 83:233-247.

77. Klamt S, Saez-Rodriguez J, Gilles ED: Structural and functional analysis of cellular networks with Cell NetAnalyzer. BMC Syst Biol 2007, 1:2.

78. Förster J, Gombert AK, Nielsen J: A functional genomics approach using metabolomics and in silico pathway analysis. Biotechnol Bioeng 2002, 79:703-712

79. Nookaew I, Meechai A, Thammarongtham C, Laoteng K, Ruanglek V, Cheevadhanarak S, Nielsen J, Bhumiratana S: Identification of flux regulation coefficients from elementary flux modes: a systems biology tool for analysis of metabolic networks. Biotechnol Bioeng 2007, 97:1535-1549.

80. Puustinen A, Finel M, Haltia T, Gennis RB, Wikstrom M: Properties of the two terminal oxidases of Escherichia coli. Biochemistry 1991, 30:3936-3942.

81. Cherry JM, Hong EL, Amundsen C, Balakrishnan R, Binkley G, Chan ET, Christie KR, Costanzo MC, Dwight SS, Engel SR, et al: Saccharomyces genome database: the genomics resource of budding yeast. Nucleic Acids Res 2012, 40:D700-D705

82. Herrgard MJ, Swainston N, Dobson P, Dunn WB, Arga KY, Arvas M, Bluthgen $\mathrm{N}$, Borger $\mathrm{S}$, Costenoble $\mathrm{R}$, Heinemann $\mathrm{M}$, et al: A consensus yeast metabolic network reconstruction obtained from a community approach to systems biology. Nat Biotechnol 2008, 26:1155-1160.

83. Kanehisa M, Goto S, Sato Y, Furumichi M, Tanabe M: KEGG for integration and interpretation of large-scale molecular data sets. Nucleic Acids Res 2012, 40:D109-D114.

84. Keseler IM, Collado-Vides J, Santos-Zavaleta A, Peralta-Gil M, Gama-Castro S, Muniz-Rascado L, Bonavides-Martinez C, Paley S, Krummenacker M, Altman T, et al: EcoCyc: a comprehensive database of Escherichia coli biology. Nucleic Acids Res 2011, 39:D583-D590.

85. Nookaew I, Jewett MC, Meechai A, Thammarongtham C, Laoteng K, Cheevadhanarak S, Nielsen J, Bhumiratana S: The genome-scale metabolic model ilN800 of Saccharomyces cerevisiae and its validation: a scaffold to query lipid metabolism. BMC Syst Biol 2008, 2:71.

86. Schomburg I, Chang A, Placzek S, Sohngen C, Rother M, Lang M, Munaretto C, Ulas S, Stelzer M, Grote A, et al: BRENDA in 2013: integrated reactions, kinetic data, enzyme function data, improved disease classification: new options and contents in BRENDA. Nucleic Acids Res 2013, 41:D764-D772.

doi:10.1186/1475-2859-12-84

Cite this article as: Gruchattka et al.: In silico profiling of Escherichia coli and Saccharomyces cerevisiae as terpenoid factories. Microbial Cell Factories 2013 12:84. 